\title{
THE CONTINUATION THEORY FOR MORSE DECOMPOSITIONS AND CONNECTION MATRICES
}

\author{
ROBERT D. FRANZOSA
}

\begin{abstract}
The continuation theory for (<-ordered) Morse decompositions and the indices defined on them - the homology index braid and the connection matrices - is established. The equivalence between <-ordered Morse decompositions and <-consistent attractor filtrations is displayed. The spaces of (<-ordered) Morse decompositions for a product parametrization of a local flow are introduced, and the local continuation of (<-ordered) Morse decompositions is obtained via the above-described equivalence and the local continuation of attractors. The homology index braid and the connection matrices of an admissible ordering of a Morse decomposition are shown to be invariant on path components of the corresponding space of <-ordered Morse decompositions. This invariance is used to prove that the collection of connection matrices of a Morse decomposition is upper semicontinuous over the space of Morse decompositions (and over the parameter space) under local continuation.
\end{abstract}

Introduction. A theorem that describes the behavior of an index under continuous change in its domain is an important component of any index theory. The Conley index is defined for isolated invariant sets in a local flow, and in [1] it is shown that for a product parameterization of a local flow, isolated invariant sets continue locally and the Conley index is invariant under the local continuation. In [10] Kurland establishes a corresponding continuation theory for the index defined on attractor-repeller pairs. It is the aim of this paper to extend these continuation results to a continuation theory for (<-ordered) Morse decompositions and the indices - the homology index braid and the connection matrices-defined on them.

Conley's and Kurland's continuation theories actually contain more than the local continuation results described above; these local results are a part of a broader global continuation scheme. In particular, in [1] Conley defines the space of isolated invariant sets for a product parameterization of a local flow and shows that this space is locally homeomorphic to the parameter space. This local homeomorphism then yields the notion of local continuation of isolated invariant sets. Next, it is shown that the Conley index is invariant on path components of the space of isolated invariant sets; this yields the invariance of the index under local continuation.

In this paper we use the same approach. We define the spaces of (<-ordered) Morse decompositions for a product parameterization of a local flow. The notion of (local) continuation of Morse decompositions and of admissible orderings of Morse

Received by the editors September 20, 1987.

1980 Mathematics Subject Classification (1985 Revision). Primary 58F35; Secondary 58F12, $34 \mathrm{C} 35$.

Key words and phrases. Conley index, Morse decomposition, connection matrix, continuation. 
decompositions is established via topological properties of these spaces. We show that the homology index braid is invariant (up to isomorphism) on path components of the spaces of <-ordered Morse decompositions. Since the connection matrices are algebraically derived from the homology index braid, it then follows that if an admissible ordering of one Morse decomposition is related by continuation to an admissible ordering of another, then the collections of connection matrices associated to these admissible orderings are equal. Therefore, the connection matrices of an admissible ordering are invariant under local continuation of the admissible ordering.

The connection matrices of a Morse decomposition (without reference to an admissible ordering) are the connection matrices of the flow ordering of the Morse decomposition. Flow orderings do not necessarily continue locally; therefore, the connection matrices of Morse decompositions are not necessarily invariant under local continuation. However, since flow orderings do continue locally to admissible orderings, and since the collection of connection matrices of an admissible ordering contains the collection of connection matrices of the flow ordering, it follows that connection matrices of Morse decompositions may be lost, but not gained, under local continuation. In this sense, the collection of connection matrices of a Morse decomposition is upper semicontinuous over the space of Morse decompositions (and over the parameter space).

$\S \S 1$ through 3 contain background material. In $\S 1$ partial orders and the algebraic connection matrix theory are reviewed. $\S 2$ covers attractor-repeller pairs, Morse decompositions, and attractor filtrations. In $\S 2$ we establish the equivalence between <-ordered Morse decompositions and attractor filtrations (thus extending similar results of Conley [1] on totally ordered Morse decompositions). $\S 3$ covers isolated invariant sets and the index theory.

In $\S 4$ the spaces of isolated invariant sets, attractor-repeller pairs, and (<ordered) Morse decompositions are defined and studied. These spaces provide the setting for the continuation theories for the various indices. Conley's [1] and Kurland's [10] continuation theories for the indices of isolated invariant sets and attractor-repeller pairs, respectively, are extended in $\S 5$ to continuation theories for the homology index braid and connection matrices. In $\S 6$ we give a simple example that shows how the connection matrix continuation theory can be used to establish the existence of connecting orbits in a parameterized family of flows.

1. Partial orders and the algebraic connection matrix theory. We summarize the main definitions and results concerning partial orders and the algebraic connection matrix theory. Further details can be found in $[\mathbf{4}, \mathbf{5}]$.

Assume throughout that $P$ is a finite indexing set containing $p$ elements. A partial order on $P$ is a relation < on the elements of $P$ satisfying:

(1) $\pi<\pi$ never holds for $\pi \in P$,

(2) $\pi<\pi^{\prime}$ and $\pi^{\prime}<\pi^{\prime \prime}$ imply $\pi<\pi^{\prime \prime}$.

A total order on $P$ is a partial order that also satisfies

(3) for each $\pi \neq \pi^{\prime} \in P$, either $\pi<\pi^{\prime}$ or $\pi^{\prime}<\pi$.

Assume throughout that $<$ is a partial order on $P$.

An extension of $<$ is a partial order $<^{\prime}$ on $P$ such that $\pi<\pi^{\prime}$ implies $\pi<^{\prime} \pi^{\prime}$. If $P^{\prime} \subset P$, then $<$ induces a partial order on $P^{\prime}$, called the restriction of $<$ to $P^{\prime}$. 
An interval in $<$ is a subset $I \subset P$ for which $\pi, \pi^{\prime} \in I$ and $\pi<\pi^{\prime \prime}<\pi^{\prime}$ imply $\pi^{\prime \prime} \in I$. The set of intervals in $<$ is denoted $I(<) . I \in I(<)$ is called an attracting interval if $\pi \in I$ and $\pi^{\prime}<\pi$ imply $\pi^{\prime} \in I$. The set of attracting intervals in $<$ is denoted $A(<) . \pi, \pi^{\prime} \in P$ are called adjacent if $\left\{\pi, \pi^{\prime}\right\} \in I(<)$.

An adjacent $n$-tuple of intervals in $<$ is an ordered collection $\left(I_{1}, \ldots, I_{n}\right)$ of mutually disjoint intervals in < satisfying:

(1) $\bigcup_{i=1}^{n} I_{i} \in I(<)$,

(2) $\pi \in I_{j}, \pi^{\prime} \in I_{k}, j<k$ imply $\pi^{\prime} \nless \pi$.

The collection of adjacent $n$-tuples of intervals in $<$ is denoted $I_{n}(<)$. Note that $I(<)=I_{1}(<)$. If $<^{\prime}$ is either an extension of $<$ or the restriction of $<$ to an interval in $<$, then $I_{n}\left(<^{\prime}\right) \subset I_{n}(<)$. If $(I, J)$ is an adjacent pair (2-tuple) of intervals, then $I \cup J$ is denoted $I J$. If $(I, J)$ and $(J, I)$ are both adjacent pairs of intervals, then $I$ and $J$ are said to be noncomparable. If $\left(I_{1}, \ldots, I_{n}\right) \in I_{n}(<)$ and $\bigcup_{i=1}^{n} I_{i}=I$, then $\left(I_{1}, \ldots, I_{n}\right)$ is called a decomposition of $I$.

DEFINITION 1.1. A graded module braid over $<$ is a collection $\mathscr{G}=\mathscr{G}(<)$ of graded modules and maps between the graded modules satisfying:

(1) for each $I \in I(<)$, there is a graded module $G(I)$,

(2) for each $(I, J) \in I_{2}(<)$, there are maps,

$$
\begin{aligned}
& i(I, I J): G(I) \rightarrow G(I J) \text { of degree } 0, \\
& p(I J, J): G(I J) \rightarrow G(J) \text { of degree } 0, \\
& \partial(J, I): G(J) \rightarrow G(I) \text { of degree }-1,
\end{aligned}
$$

that satisfy:

(a) $\cdots \rightarrow G(I) \stackrel{i}{\rightarrow} G(I J) \stackrel{p}{\rightarrow} G(J) \stackrel{\partial}{\rightarrow} G(I) \rightarrow \cdots$ is exact,

(b) if $I$ and $J$ are noncomparable, then $p(J I, I) i(I, I J)=$ id $\mid G(I)$,

(c) if $(I, J, K) \in I_{3}(<)$, then the following braid diagram commutes.

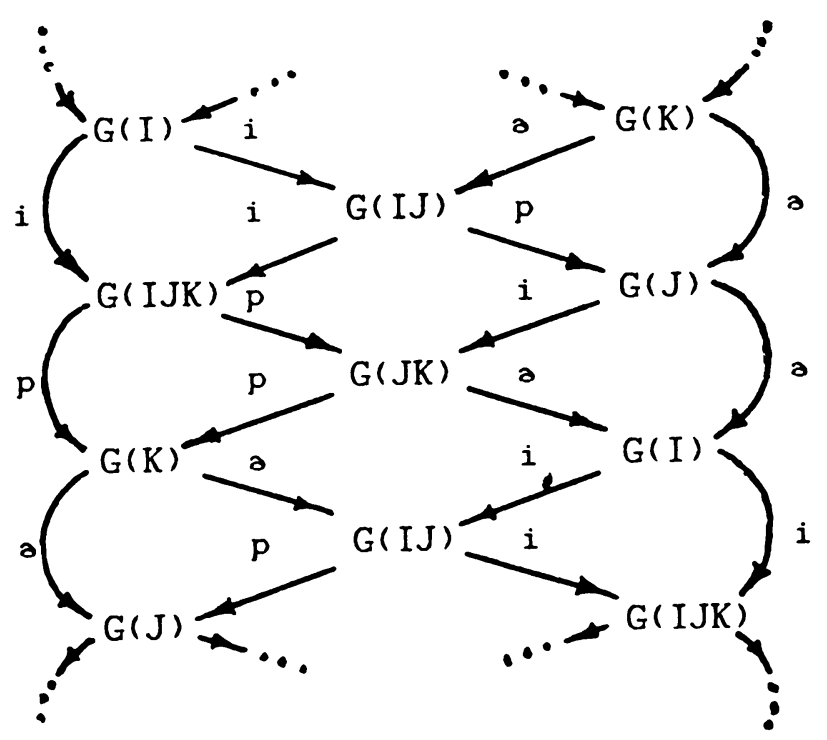


The homology index braid of an admissible ordering of a Morse decomposition (see $[\mathbf{4}, \mathbf{5}]$ and $\S 3$ below) is an example of a graded module braid.

Assume that $\mathscr{G}$ and $\mathscr{G}^{\prime}$ are graded module braids over $<$.

DEFinition 1.2. A. A map $\theta$ from $\mathscr{G}$ to $\mathscr{G}^{\prime}$, denoted $\theta: \mathscr{G} \rightarrow \mathscr{G}^{\prime}$, is a collection $\{\theta(I)\}_{I \in I(<)}$ of module homomorphisms $\theta(I): G(I) \rightarrow G^{\prime}(I)$ such that the following diagram commutes for each $(I, J) \in I_{2}(<)$ :

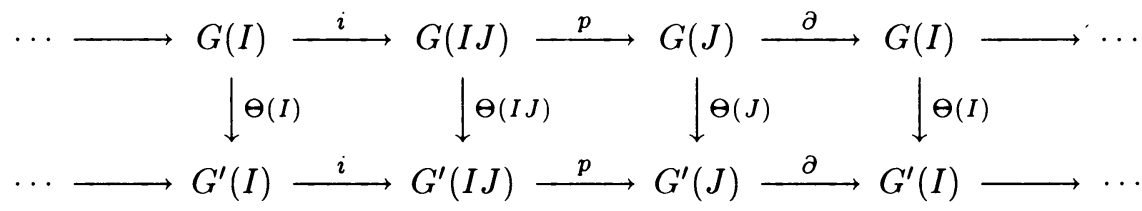

B. If, furthermore, $\theta(I)$ is an isomorphism for each $I \in I(<)$, then $\theta$ is called an isomorphism and $\mathscr{G}$ and $\mathscr{G}^{\prime}$ are said to be isomorphic.

Let $<$ ' be either an extension of $<$ or the restriction of $<$ to an interval $I \in I(<)$. If $\mathscr{G}$ is a graded module braid over $<$, then $\mathscr{G}$ can be restricted to a graded module braid $\mathscr{G} \mid<^{\prime}$ over $<^{\prime}$. Specifically, let $\mathscr{G} \mid<^{\prime}$ be the collection of the graded modules $G(I)$, for $I \in I\left(<^{\prime}\right) \subset I(<)$, and the maps $i(I, I J), p(I J, J)$, and $\partial(J, I)$, for $(I, J) \in I_{2}\left(<^{\prime}\right) \subset I_{2}(<)$. It is not difficult to see that $\mathscr{G} \mid<^{\prime}$ is a graded module braid over $<^{\prime}$.

Let $C=\{C \Delta(\pi)\}_{\pi \in P}$ be a collection of graded modules. If $I \subset P$, then a map $\Delta: \bigoplus_{\pi \in I} C \Delta(\pi) \rightarrow \bigoplus_{\pi \in I} C \Delta(\pi)$ can be regarded as a matrix

$$
\left(\begin{array}{c}
\vdots \\
\cdots \Delta_{\pi, \pi^{\prime}} \cdots \\
\vdots
\end{array}\right)_{\pi, \pi^{\prime} \in I}:\left(\begin{array}{c}
\vdots \\
C \Delta(\pi) \\
\vdots
\end{array}\right)_{\pi \in I} \rightarrow\left(\begin{array}{c}
\vdots \\
C \Delta(\pi) \\
\vdots
\end{array}\right)_{\pi \in I}
$$

where each $\Delta_{\pi, \pi^{\prime}}$ is a map from $C \Delta\left(\pi^{\prime}\right)$ to $C \Delta(\pi)$.

DEFINITION 1.3. A. $\Delta$ is upper triangular if $\Delta_{\pi, \pi^{\prime}} \neq 0$ implies $\pi \leq \pi^{\prime}$.

B. $\Delta$ is strictly upper triangular if $\Delta_{\pi, \pi^{\prime}} \neq 0$ implies $\pi<\pi^{\prime}$.

C. $\Delta$ is a boundary map if each $\Delta_{\pi, \pi^{\prime}}$ is of degree -1 and $\Delta^{2}=0$.

Assume that $\Delta: \bigoplus_{\pi \in P} C \Delta(\pi) \rightarrow \bigoplus_{\pi \in P} C \Delta(\pi)$ is an upper triangular boundary map. It is shown in [5] that $\Delta$ naturally defines a graded module braid over $<$, denoted $\mathscr{H} \Delta(<)$.

DEFINITION 1.4. Given $\mathscr{G}$, a graded module braid over $<$, and $C=\{C \Delta(\pi)\}_{\pi \in P}$, a collection of graded modules, let $\Delta: \bigoplus_{\pi \in P} C \Delta(\pi) \rightarrow \bigoplus_{\pi \in P} C \Delta(\pi)$ be an upper triangular boundary map. Then,

A. if $\mathscr{H} \Delta$ is isomorphic to $\mathscr{G}$, then $\Delta$ is called a $C$-connection matrix of $\mathscr{G}$,

B. if, furthermore, $C \Delta(\pi)$ is isomorphic to $G(\pi)$ for each $\pi \in P$, then $\Delta$ is called a connection matrix of $\mathscr{G}$.

The collection of $C$-connection matrices and connection matrices of $\mathscr{G}$ are denoted by $\mathscr{C} \mathscr{M}(\mathscr{G}, C)$ and $\mathscr{C} \mathscr{M}(\mathscr{G})$, respectively.

Let $C=\{C \Delta(\pi)\}_{\pi \in P}$ be a collection of graded modules. The following proposition describes the relationships between $(C$-)connection matrices under isomorphism of graded module braids and under extension of the underlying partial order.

PROPOSITION 1.5. A. If $\mathscr{G}$ and $\mathscr{G}^{\prime}$ are isomorphic graded module braids, then

$$
\mathscr{C} \mathscr{M}(\mathscr{G}, C)=\mathscr{C} \mathscr{M}\left(\mathscr{G}^{\prime}, C\right) \text { and } \mathscr{C} \mathscr{M}(\mathscr{G})=\mathscr{C} \mathscr{M}\left(\mathscr{G}^{\prime}\right) \text {. }
$$


B. If $\mathscr{G}$ is defined over a partial order having extension $<^{\prime}$, then $\mathscr{C} \mathscr{M}(\mathscr{G}, C) \subset$ $\mathscr{C} \mathscr{M}\left(\mathscr{G} \mid<^{\prime}, C\right)$ and $\mathscr{C} \mathscr{M}(\mathscr{G}) \subset \mathscr{C} \mathscr{M}\left(\mathscr{G} \mid<^{\prime}\right)$.

2. Attractor-repeller pairs, Morse decompositions, and attractor filtrations. Some of the following results concerning attractor-repeller pairs and Morse decompositions are stated without proof. Further details can be found in [1, 4].

Let $\Gamma$ be a Hausdorff topological space with a flow, and assume $S$ is compact invariant in $\Gamma$. If $S_{1}, S_{2}$ are invariant subsets of $S$, then $C\left(S_{2}, S_{1} ; S\right):=\{x \in$ $\left.S \mid \omega^{*}(x) \subset S_{2}, \omega(x) \subset S_{1}\right\}$ is the set of orbits connecting $S_{2}$ to $S_{1}$ in $S$. This set is usually denoted $C\left(S_{2}, S_{1}\right)$.

If $A \subset S$ is the $\omega$-limit set of a neighborhood $U$ of itself in $S$, then $A$ is an attractor in $S$ and $U$ is an attracting neighborhood of $A$. Similarly a repeller in $S$ is the $\omega^{*}$-limit set of a (repelling) neighborhood of itself in $S$.

If $A$ is an attractor in $S$, then $A^{*}:=\{x \in S \mid \omega(x) \cap A=\varnothing\}$ is a repeller in $S$, called the repeller complementary to $A$ in $S$, and $S$ decomposes into the union $S=A \cup C\left(A^{*}, A\right) \cup A^{*}$. If $A_{1}, A_{2}$ are attractors in $S$ with attracting neighborhoods $U_{1}, U_{2}$, respectively, then $A_{1} \cup A_{2}, A_{1} \cap A_{2}$ are attractors in $S$ with attracting neighborhoods $U_{1} \cup U_{2}, U_{1} \cap U_{2}$ and complementary repellers $A_{1}^{*} \cap A_{2}^{*}, A_{1}^{*} \cup A_{2}^{*}$, respectively.

The following result due to Conley [1] plays an important role in the continuation theories for attractor-repeller pairs and Morse decompositions. It implies that if $\hat{S}$ is a compact invariant set near $S$, then near any attractor in $S$, there is a corresponding attractor in $\hat{S}$.

PROPOSITION 2.1. Let $Y_{0}$ be a $\Gamma$-neighborhood of an attractor in $S$. If $Y_{0} \cap S$ is an attracting neighborhood of the attractor, then there exists a $\Gamma$-open neighborhood $Y_{1}$ of $S$ such that if $\hat{S} \subset Y_{1}$ is compact invariant, then $Y_{0} \cap \hat{S}$ is an attracting neighborhood of some (possibly empty) attractor in $\hat{S}$.

The decomposition of $S$ by an attractor-repeller pair is generalized by the Morse decompositions of $S$; specifically,

DEFINITION 2.2. A (<-ordered) Morse decomposition of $S$ is a collection $M=$ $M(S)=\{M(\pi)\}_{\pi \in P}$ of mutually disjoint compact invariant subsets of $S$ such that if $\gamma \in S \backslash \bigcup_{\pi \in P} M(\pi)$, then there exist $\pi<\pi^{\prime}$ with $\gamma \in C\left(M\left(\pi^{\prime}\right), M(\pi)\right)$.

Since a collection of sets $M=\{M(\pi)\}_{\pi \in P}$ can be a Morse decomposition of more than one invariant set, the structures (including the indices) associated to a Morse decomposition of an invariant set $S$ are defined relative to $S$. However, for notational convenience, we usually omit reference to $S$ in the discussions of the structures.

Assume that $M=\{M(\pi)\}_{\pi \in P}$ is a <-ordered Morse decomposition of $S$. The partial order < on $P$ induces an obvious partial order on $M$; it also is denoted $<$ and is called an admissible ordering of $M$. The flow defines an "extremal" admissible ordering of $M$, called the flow ordering of $M$, denoted $<_{F}$, and such that $M(\pi)<_{F} M\left(\pi^{\prime}\right)$ if and only if there exists a sequence of distinct elements of $P: \pi=\pi_{0}, \ldots, \pi_{n}=\pi^{\prime}$, with $C\left(M\left(\pi_{j}\right), M\left(\pi_{j-1}\right)\right) \neq \varnothing$ for each $j=1, \ldots, n$. Every admissible ordering of $M$ is an extension of $<_{F}$. 
For each $I \in I(<)$, let

$$
M(I)=\left(\bigcup_{\pi \in I} M(\pi)\right) \cup\left(\bigcup_{\pi, \pi^{\prime} \in I} C\left(M\left(\pi^{\prime}\right), M(\pi)\right)\right) .
$$

$M(I)$ is called a Morse set of the admissible ordering $<$ (or a Morse set of the Morse decomposition $M)$. The collection $\{M(I) \mid I \in I(<)\}$ is denoted $M S(<)$. Since $<$ is an extension of $<_{F}$, it follows that $M S(<) \subset M S\left(<_{F}\right)$. Because of this, $M S\left(<_{F}\right)$ is also denoted $M S(M)$.

If $(I, J) \in I_{2}(<)$, then $(M(I), M(J))$ is an attractor-repeller pair in $M(I J)$; in particular, if $I$ is an attracting interval in <, then $M(I)$ is an attractor in $S$ with complementary repeller $M(P \backslash I)$.

The collection of attractors in $S, A F(<):=\{M(I) \mid I \in A(<)\}$, is called the attractor filtration of the admissible ordering $<$ of $M$. More generally,

DEFINITION 2.3. A. An attractor filtration in $S$ is a finite collection $A F$ of attractors in $S$ such that

(1) $\varnothing, S \in A F$,

(2) if $A_{1}, A_{2} \in A F$, then $A_{1} \cup A_{2}, A_{1} \cap A_{2} \in A F$.

B. An attractor filtration is called <-consistent if it can be indexed by $A(<)$ so that $A_{I_{1}} \cup A_{I_{2}}=A_{I_{1} \cup I_{2}}$ and $A_{I_{1}} \cap A_{I_{2}}=A_{I_{1} \cap I_{2}}$ for every $I_{1}, I_{2} \in A(<)$.

Thus, a <-ordered Morse decomposition defines a <-consistent attractor filtration $A F(<)$. Below, we show that this correspondence is one-to-one; i.e., that each $<-$ consistent attractor filtration determines a <-ordered Morse decomposition. In $\S 4$, this equivalence plays an important role in the continuation theory for Morse decompositions because the local continuation of attractors (Proposition 2.1) yields the local continuation of attractor filtrations and therefore (by this equivalence) the local continuation of Morse decompositions.

In [1] Conley exhibits the equivalence between <-ordered Morse decompositions and $<$-consistent attractor filtrations for total orders $<$. We extend those results to establish the equivalence for general partial orders.

THEOREM 2.4. Let $A F=\left\{A_{I}\right\}_{I \in A(<)}$ be a <-consistent attractor filtration in $S$. There exists a <-ordered Morse decomposition $M$ of $S$ such that $A_{I}=M(I)$ for each $I \in A(<)$ (and therefore $A F=A F(<)$ ).

To prove Theorem 2.4 we need the following

Lemma 2.5. For $i=1,2$, assume $K_{i} \in A(<)$ and $\left(L_{i}, J\right)$ is a decomposition of $K_{i}$. Then $L_{i} \in A(<)$ and $A_{K_{1}} \cap A_{L_{1}}^{*}=A_{K_{2}} \cap A_{L_{2}}^{*}$.

ProOF. It is easy to see that $L_{i} \in A(<)$. Let $L=L_{1} \cap L_{2}, K=K_{1} \cap K_{2}$. $L, K \in A(<)$, and $(L, J)$ is a decomposition of $K$. It is enough to show that $A_{K} \cap A_{L}^{*}=A_{K_{1}} \cap A_{L_{1}}^{*}$. Note that $K_{1}=K \cup L_{1}$ and $K \cap L_{1}=L$; therefore, $A_{K_{1}}=A_{K} \cup A_{L_{1}}$ and $A_{K} \cap A_{L_{1}}=A_{L}$. Now,

$$
\begin{aligned}
A_{K_{1}} \cap A_{L_{1}}^{*} & =\left(A_{K} \cup A_{L_{1}}\right) \cap A_{L_{1}}^{*}=A_{K} \cap A_{L_{1}}^{*} \\
& =A_{K} \cap\left(A_{K}^{*} \cup A_{L_{1}}^{*}\right)=A_{K} \cap\left(A_{K} \cap A_{L_{1}}\right)^{*}=A_{K} \cap A_{L}^{*} .
\end{aligned}
$$

PROOF OF THEOREM 2.4. Order the elements of $P: \pi_{1}, \ldots, \pi_{p}$, such that $\pi_{i}<\pi_{j}$ implies $i<j$. Let $I_{0}=\varnothing$, and for each $j=1, \ldots, p$, let $I_{j}=\left\{\pi_{i} \mid 1 \leq i \leq j\right\}$. 
Clearly, $I_{j} \in A(<)$ and $A_{I_{j-1}} \subset A_{I_{j}}$. For $j=1, \ldots, p$, define $M\left(\pi_{j}\right)=A_{I_{j}} \cap A_{I_{j-1}}^{*}$. By Conley [1] $, M:=\{M(\pi)\}_{\pi \in P}$ is a totally ordered Morse decomposition of $S$. We claim that $M$ is <-ordered and $M(I)=A_{I}$ for each $I \in A(<)$.

We begin by showing that if $\pi \in I$, then $M(\pi) \subset A_{I}$, otherwise $M(\pi) \cap A_{I}=\varnothing$.

Assume $\pi \in I$. Define $K=\{\alpha \in P \mid \alpha \leq \pi\}$ and $L=K \backslash \pi . \quad K \in A(<)$, $(L, \pi)$ is a decomposition of $K$, and $K \subset I$. It follows that $A_{K} \subset A_{I}$. We claim that $M(\pi) \subset A_{K}$, implying $M(\pi) \subset A_{I}$. Let $\pi=\pi_{j} . I_{j} \in A(<)$, and $\left(I_{j-1}, \pi\right)$ is a decomposition of $I_{j}$. The lemma implies $A_{I_{j}} \cap A_{I_{j-1}}^{*}=A_{K} \cap A_{L}^{*}$. Thus, $M(\pi) \subset A_{K}$.

Assume $\pi \notin I$. Define $K=\{\alpha \in P \mid \pi \nless \alpha\}$ and $L=K \backslash \pi . \quad K \in A(<)$, $(L, \pi)$ is a decomposition of $K$, and $I \subset L$. It follows that $A_{I} \subset A_{L}$. We claim that $M(\pi) \cap A_{L}=\varnothing$, implying $M(\pi) \cap A_{I}=\varnothing$. As above, if $\pi=\pi_{j}$, then the lemma implies $A_{I_{j}} \cap A_{I_{j-1}}^{*}=A_{K} \cap A_{L}^{*}$. It follows that $M(\pi) \subset A_{L}^{*}$, and therefore $M(\pi) \cap A_{L}=\varnothing$.

We show that $M$ is <-ordered. Assume $\gamma \in S \backslash \bigcup_{\pi \in P} M(\pi)$. Since $M$ is a Morse decomposition, there exist $\pi \neq \pi^{\prime}$ with $\gamma \in C\left(M\left(\pi^{\prime}\right), M(\pi)\right)$. We claim that $\pi<\pi^{\prime}$. Let $I=\left\{\alpha \in P \mid \alpha \leq \pi^{\prime}\right\}$. We prove that $\pi \in I$. Then since $\pi \neq \pi^{\prime}$, it follows that $\pi<\pi^{\prime} . I \in A(<)$, and $\pi^{\prime} \in I$ implies that $M\left(\pi^{\prime}\right) \subset A_{I}$. So $\omega^{*}(\gamma) \subset A_{I}$, and since $A_{I}$ is an attractor, it follows that $\gamma \in A_{I}$ and $\omega(\gamma) \subset A_{I}$. Therefore $M(\pi) \cap A_{I} \neq \varnothing$, implying $\pi \in I$.

We show that $M(I)=A_{I}$ for each $I \in A(<) . M(\pi) \subset A_{I}$ for each $\pi \in I$, and if $\pi, \pi^{\prime} \in I$ and $\gamma \in C\left(M\left(\pi^{\prime}\right), M(\pi)\right)$, then, as in the proof that $\pi<\pi^{\prime}$, it follows that $\gamma \in A_{I}$. Thus, $M(I) \subset A_{I}$. Now, suppose $\gamma \in A_{I}$. Then if $\gamma \in M(\pi)$ for some $\pi \in P$, then $\pi \in I$, and therefore $\gamma \in M(I)$. If $\gamma \in C\left(M\left(\pi^{\prime}\right), M(\pi)\right)$ for $\pi, \pi^{\prime} \in P$, then both $M(\pi) \cap A_{I}$ and $M\left(\pi^{\prime}\right) \cap A_{I}$ are nonempty, implying that $\pi, \pi^{\prime} \in I$. It follows that $\gamma \in M(I)$, and therefore $A_{I} \subset M(I)$.

Note that Lemma 2.5 implies that the Morse decomposition in Theorem 2.4 is independent of the choice of the ordering $\pi_{1}, \ldots, \pi_{p}$ of the elements of $P$. The Morse decomposition in Theorem 2.4 is called the <-ordered Morse decomposition defined by the <-consistent attractor filtration $A F$. The above result states that $A F$ equals $A F(<)$, the attractor filtration of the admissible ordering $<$ of the Morse decomposition defined by $A F$. It is easy to see that if $M$ is a <-ordered Morse decomposition, then $M$ equals the Morse decomposition defined by $A F(<)$, the attractor filtration of the admissible ordering $<$ of $M$.

It is not difficult to prove that an attractor filtration is <-consistent for some partial order on some set $P$, and therefore every attractor filtration defines a Morse decomposition. We make no use of this result and therefore we do not pursue it further.

3. Isolated invariant sets and the index theory. In this section we outline the index theory for isolated invariant sets, attractor-repeller pairs in isolated invariant sets, and Morse decompositions of isolated invariant sets. Further details on these topics can be found in $[1-5,8-10,18,19]$.

Assume that $X$ is a locally compact metric local flow in $\Gamma$. Let $S \subset X$ be a compact invariant set. If $S$ is the maximal invariant set in a compact neighborhood $N$ of itself in $X$, then $S$ is called an $(X$-)isolated invariant set and $N$ is called an $(X$-)isolating neighborhood of $S$. 
If $S_{1}$ and $S_{2}$ are isolated invariant sets with isolating neighborhoods $N_{1}$ and $N_{2}$, respectively, then $S_{1} \cap S_{2}$ is an isolated invariant set with isolating neighborhood $N_{1} \cap N_{2}$. It does not follow that $S_{1} \cup S_{2}$ is an isolated invariant set nor that $N_{1} \cup N_{2}$ is an isolating neighborhood.

Assume that $S$ is an isolated invariant set. The following propositions establish that an attractor in $S$, its complementary repeller in $S$, and the Morse sets of a Morse decomposition of $S$ are isolated invariant sets. The other implications in these propositions play an important role in the continuation theories for attractor-repeller pairs and Morse decompositions. The straightforward proofs of these propositions are left to the reader (see [3]).

Proposition 3.1. If $\left(A, A^{*}\right)$ is an attractor-repeller pair in $S$, then $A$ and $A^{*}$ are $X$-isolated invariant sets. Furthermore, if $N$ and $U$ are isolating neighborhoods of $S$ and $A$, respectively, and $U \cap S$ is an attracting neighborhood of $A$ in $S$, then $\operatorname{cl}_{X}(N \backslash U)$ is an $X$-isolating neighborhood of $A^{*}$.

If $A$ is an attractor in $S$, then an $(X$-)isolating neighborhood of $A$ whose intersection with $S$ is an attracting neighborhood of $A$ in $S$ is called an (X-)isolating $S$-attracting neighborhood of $A$.

Proposition 3.2. Let $A_{1}$ and $A_{2}$ be attractors in $S$ with $X$-isolating $S$ attracting neighborhoods $U_{1}$ and $U_{2}$, respectively. Then $U_{1} \cap U_{2}$ is an $X$-isolating $S$-attracting neighborhood of $A_{1} \cap A_{2}$, and if $U_{1}$ and $U_{2}$ are contained in some isolating neighborhood of $S$, then $U_{1} \cup U_{2}$ is an $X$-isolating $S$-attracting neighborhood of $A_{1} \cup A_{2}$.

PROPOSITION 3.3. If $M$ is a <-ordered Morse decomposition of $S$, then the associated Morse sets are isolated invariant sets. Furthermore, if $U_{I}$ is an $X$ isolating $S$-attracting neighborhood of $M(I)$ for each $I \in A(<)$ and $(I, J)$ is a decomposition of $K \in A(<)$, then $\operatorname{cl}_{X}\left(U_{K} \backslash U_{I}\right)$ is an $X$-isolating neighborhood of $M(J)$.

We now proceed with a description of the index theory.

Given $Z \subset Y \subset \Gamma$, we call $Z$ positively invariant relative to $Y$ if $\gamma \in Z$ and $\gamma \cdot[0, t] \subset Y$ together imply that $\gamma \cdot[0, t] \subset Z$. By a compact pair $\left(N_{1}, N_{0}\right)$, we mean an ordered pair of compact spaces with $N_{0} \subset N_{1}$.

DEFINITION 3.4. A compact pair $\left(N_{1}, N_{0}\right)$ in $X$ is called an $(X$-)index pair for $S$ if

(1) $S \subset \operatorname{int}_{X}\left(N_{1} \backslash N_{0}\right)$,

(2) $S$ is the maximal invariant set in $\operatorname{cl}_{X}\left(N_{1} \backslash N_{0}\right)$,

(3) $N_{0}$ is positively invariant relative to $N_{1}$,

(4) $\gamma \in N_{1}$ and $\gamma \cdot R^{+} \not \subset N_{1}$ imply there exists $t \geq 0$ such that $\gamma \cdot[0, t] \subset N_{1}$ and $\gamma \cdot t \in N_{0}$.

In [1] Conley proves the existence of index pairs for isolated invariant sets. Furthermore, he proves that the homotopy type of the pointed quotient space $N_{1} / N_{0}$ is independent of the index pair. Thus, a homotopy type of a pointed space is associated to the isolated invariant set $S$. It is denoted $h(S)$ and called the Conley index of $S$ (relative to $X$ ).

The work on indices in this paper is done on the homology level. Singular homology with coefficients in a module $G$ over a PID is used, and $H_{*}(h(S) ; G)$ 
is called the homology index of $S$ (with coefficients in $G$ and relative to $X$ ). For notational simplicity, we denote this by $H(S)$. For the remainder of the paper, we omit reference to $X$ and $G$ in the various indices. However, it should be kept in mind that all of the indices depend on the local flow $X$ and on the coefficient module $G$, and furthermore, in the case of the indices associated to a Morse decomposition $M$, there is also dependence on the invariant set of which $M$ is a Morse decomposition.

Let $\left(A, A^{*}\right)$ be an attractor-repeller pair in $S$. If $N_{0} \subset N_{1} \subset N_{2}$ are such that $\left(N_{1}, N_{0}\right)$ and $\left(N_{2}, N_{0}\right)$ are index pairs for $A$ and $S$, respectively, then $\left(N_{2}, N_{1}\right)$ is an index pair for $A^{*}$. The triple $\left(N_{2}, N_{1}, N_{0}\right)$ is called an index triple for $\left(A, A^{*}\right)$ in $S$; it defines a sequence of maps on quotient spaces, $N_{1} / N_{0} \stackrel{i}{\rightarrow} N_{2} / N_{0} \stackrel{p}{\rightarrow} N_{2} / N_{1}$. Passing to homology, there is an exact sequence,

$$
\cdots \rightarrow H_{*}\left(N_{1} / N_{0}\right) \stackrel{i}{\rightarrow} H_{*}\left(N_{2} / N_{0}\right) \stackrel{p}{\rightarrow} H_{*}\left(N_{2} / N_{1}\right) \stackrel{\partial}{\rightarrow} H_{*}\left(N_{1} / N_{0}\right) \stackrel{i}{\rightarrow} \cdots .
$$

This sequence is independent of the choice of index triple for $\left(A, A^{*}\right)$ in $S$ and therefore defines a sequence, called the homology index sequence for $\left(A, A^{*}\right)$ in $S$,

$$
\cdots \rightarrow H(A) \stackrel{i}{\rightarrow} H(S) \stackrel{p}{\rightarrow} H\left(A^{*}\right) \stackrel{\partial}{\rightarrow} H(A) \stackrel{i}{\rightarrow} \cdots .
$$

Assume $M$ is a <-ordered Morse decomposition of $S$. The homology index $H(M(I))$ is defined for each Morse set of the admissible ordering < (for simplicity it is denoted $H(I))$, and for each $(I, J) \in I_{2}(<)$, there is a sequence

$$
\cdots \rightarrow H(I) \stackrel{i}{\rightarrow} H(I J) \stackrel{p}{\rightarrow} H(J) \stackrel{\partial}{\rightarrow} H(I) \stackrel{i}{\rightarrow} \cdots .
$$

The collection of the $H(I)$, for all $I \in I(<)$, and the maps $i, p, \partial$, for all $(I, J) \in$ $I_{2}(<)$, is a graded module braid over $<$. It is called the homology index braid of the admissible ordering $<$ of $M$ and is denoted $\mathscr{H}(<)$. The homology index braid of the flow ordering of $M$ is called the homology index braid of $M$ and is denoted $\mathscr{H}(M)$. It is easy to see that $\mathscr{H}(<) \subset \mathscr{H}(M)$.

Let $C=\{C \Delta(\pi)\}_{\pi \in P}$ be a collection of graded modules. $\mathscr{C} \mathscr{M}(\mathscr{H}(<), C)$ is called the collection of $C$-connection matrices of the admissible ordering $<$ of $M$ and is denoted $\mathscr{C} \mathscr{M}(<, C) . \mathscr{C} \mathscr{M}(\mathscr{H}(M), C)$ is called the collection of $C$-connection matrices of $M$ and is denoted $\mathscr{C} \mathscr{M}(M, C)$. $\mathscr{C} \mathscr{M}(<)$ and $\mathscr{C} \mathscr{M}(M)$, the collections of connection matrices, are similarly defined. By Proposition 1.5.B, $\mathscr{C} \mathscr{M}(M, C) \subset$ $\mathscr{C} \mathscr{M}(<, C)$ and $\mathscr{C} \mathscr{M}(M) \subset \mathscr{C} \mathscr{M}(<)$. It is shown in [5] that if each $C \Delta(\pi)$ is a free chain complex with homology isomorphic to $H(\pi)$, then $\mathscr{\mathscr { M }}(<, C)$ and $\mathscr{C} \mathscr{M}(M, C)$ are nonempty. In particular, if $H(\pi)$ is free for each $\pi \in P$ (e.g., if the coefficient module $G$ is a field), then $\mathscr{C} \mathscr{M}(M)$ and $\mathscr{C} \mathscr{M}(<)$ are nonempty.

4. The continuation theory for Morse decompositions. We define a product parameterization of a local flow and the spaces of isolated invariant sets, attractor-repeller pairs, and Morse decompositions for the product parameterization. Through topological properties of these spaces the continuation theory for Morse decompositions is established. These spaces also provide the setting for the continuation theories for homology index braids and connection matrices.

Assume that $Z$ is a locally compact space and $\Lambda$ is locally path connected.

DEFINITION 4.1. A product parameterization of the local flow $X \subset \Gamma$ is a homeomorphism $\phi: Z \times \Lambda \rightarrow X$ such that for each $\lambda \in \Lambda, \phi(Z \times\{\lambda\})$ is a local flow. 
Let $\phi: Z \times \Lambda \rightarrow X$ be a product parameterization of the local flow $X$. We denote the restriction $\phi \mid(Z \times\{\lambda\})$ by $\phi_{\lambda}$ and its image by $X_{\lambda}$.

DEFINITION 4.2. The space of isolated invariant sets for the product parameterization is the set

$$
\mathscr{S}=\mathscr{S}(\phi)=\left\{\left(S_{\lambda}, X_{\lambda}\right) \mid S_{\lambda} \text { is an isolated invariant set in } X_{\lambda}\right\} .
$$

To simplify notation, we write $S_{\lambda}$ instead of $\left(S_{\lambda}, X_{\lambda}\right)$. However, it should be noted that $\mathscr{S}$ is the setting for the continuation theory for the Conley index, and the Conley index depends on both the invariant set $S_{\lambda}$ and the local flow $X_{\lambda}$.

Given $N$, compact and contained in $Z$, let

$$
\Lambda(N)=\left\{\lambda \in \Lambda \mid \phi_{\lambda}(N) \text { is an isolating neighborhood in } X_{\lambda}\right\} .
$$

Define $\sigma_{N}: \Lambda(N) \rightarrow \mathscr{S}, \sigma_{N}(\lambda)=S_{\lambda}$ where $S_{\lambda}$ is the maximal invariant set in $\phi_{\lambda}(N)$.

Proposition 4.3 (SEE [1]). Let $N_{1}, N_{2} \subset Z$ be compact. Assume $\lambda \in$ $\Lambda\left(N_{1}\right) \cap \Lambda\left(N_{2}\right)$ and the maximal invariant sets in $\phi_{\lambda}\left(N_{1}\right)$ and $\phi_{\lambda}\left(N_{2}\right)$ are equal. Then there exists $W$, a neighborhood of $\lambda$ contained in $\Lambda\left(N_{1}\right) \cap \Lambda\left(N_{2}\right)$, such that $\mu \in W$ implies $\phi_{\mu}\left(N_{1}\right)$ and $\phi_{\mu}\left(N_{2}\right)$ have the same maximal irvariant set.

As a consequence of Proposition 4.3, we have

Proposition 4.4 (SEE [1]). A. $\Lambda(N)$ is open in $\Lambda$ for each compact $N \subset Z$.

B. The collection $\mathscr{B}=\left\{\sigma_{N}(U) \mid N \subset Z\right.$ is compact and $U \subset \Lambda(N)$ is open $\}$ is a basis for a topology on $\mathscr{P}$.

Assume that $\mathscr{P}$ has the topology induced by the basis $\mathscr{B}$ described above. Define $\pi_{, j}: \mathscr{P} \rightarrow \Lambda, \pi_{\text {, }}\left(S_{\lambda}\right)=\lambda$.

PROPOSITION 4.5 (SEE $[\mathbf{1}])$. $\pi_{. \nsucc}$ is a surjective local homeomorphism; in particular, for each $N$ compact in $Z, \pi_{-N} \mid \sigma_{N}(\Lambda(N))$ is a homeomorphism with inverse $\sigma_{N}$.

Thus, if $S_{\lambda}$ is an isolated invariant set in $X_{\lambda}$, then for $\mu$ near $\lambda, X_{\mu}$ contains an isolated invariant set $S_{\mu}$ near $S_{\lambda}$. On a global basis, we say that two invariant sets, $S_{\lambda}$ in $X_{\lambda}$ and $S_{\mu}$ in $X_{\mu}$, are related by continuation or are continuations of each other if the corresponding points in $\mathscr{P}$ lie in the same (path) component. The invariance of the Conley index under continuation (see [1] and §5) states that the index is independent of the component of $\mathcal{P}$.

Next, we present the continuation theory for attractor-repeller pairs. We define the space of attractor-repeller pairs for the product parameterization $\phi$ to be the set

$$
\mathscr{A} \mathscr{R}=\mathscr{A} \mathscr{R}(\phi)=\left\{\left(A_{\lambda}, A_{\lambda}^{*}, S_{\lambda}\right) \in \mathscr{S}(\phi) \times \mathscr{P}(\phi) \times \mathscr{S}(\phi) \mid\right.
$$

$\left(A_{\lambda}, A_{\lambda}^{*}\right)$ is an attractor-repeller pair in $\left.S_{\lambda}\right\}$

with the topology inherited as a subspace of $\mathscr{P}(\phi) \times \mathscr{S}(\phi) \times \mathscr{S}(\phi)$.

In [10] Kurland defines a space of attractor-repeller pairs for a product parameterization. His construction is somewhat different from ours; however, the resulting spaces are essentially identical.

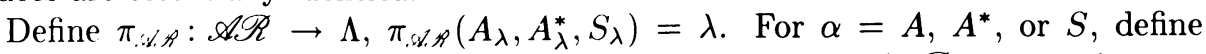
$\mathscr{P}_{\alpha}: \mathscr{A} \mathscr{R} \rightarrow \mathscr{S}, \mathscr{P}_{\alpha}\left(A_{\lambda}, A_{\lambda}^{*}, S_{\lambda}\right)=\alpha_{\lambda}$. Clearly, $\pi_{\mathscr{H}, \mathscr{H}}$ and each $\mathscr{P}_{\alpha}$ are continuous. 
For $\alpha=A, A^{*}$, or $S$ consider

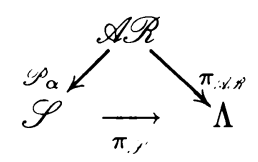

Diagram (4.1) commutes. We claim that each map in diagram (4.1) is a surjective local homeomorphism. $\pi_{. j}$ is a surjective local homeomorphism by Proposition 4.5. We prove that $\pi_{\mathscr{2}, h}$ is a surjective local homeomorphism in Theorem 4.8 , below. Kurland proves a corresponding result in [10].

The idea is to prove that if $\left(A_{\lambda}, A_{\lambda}^{*}\right)$ is an attractor-repeller pair in $S_{\lambda} \subset X_{\lambda}$ and $\mu$ is near $\lambda$, then the invariant sets $A_{\lambda}, A_{\lambda}^{*}, S_{\lambda}$, respectively, continue to invariant sets $A_{\mu}, A_{\mu}^{*}, S_{\mu} \subset X_{\mu}$ such that $\left(A_{\mu}, A_{\mu}^{*}\right)$ is an attractor-repeller pair in $S_{\mu}$. Proposition 4.6, below, implies that $A_{\mu}$, the continuation of $A_{\lambda}$, is an attractor in $S_{\mu}$, the continuation of $S_{\lambda}$. Proposition 4.7, below, then implies that the repeller complementary to $A_{\mu}$ in $S_{\mu}$ is the continuation of $A_{\lambda}^{*}$.

Using Propositions 4.6 and 4.7, we then prove that the maps in diagram (4.1) are surjective local homeomorphisms.

Proposition 4.6. Assume $\left(A_{\lambda}, A_{\lambda}^{*}\right)$ is an attractor-repeller pair in $S_{\lambda} \subset X_{\lambda}$. Let $V$ and $U$ be such that $\phi_{\lambda}(V)$ is an $X_{\lambda}$-isolating neighborhood of $S_{\lambda}$ and $\phi_{\lambda}(U)$ is an $X_{\lambda}$-isolating $S_{\lambda}$-attracting neighborhood of $A_{\lambda}$. Then there exists $W$, an open neighborhood of $\lambda$ contained in $\Lambda(V) \cap \Lambda(U)$, such that $\mu \in W$ implies

(1) if $\sigma_{U}(\mu)=A_{\mu}$ and $\sigma_{V}(\mu)=S_{\mu}$, then $A_{\mu}$ is an attractor in $S_{\mu}$,

(2) $\phi_{\mu}(U)$ is an $X_{\mu}$-isolating $S_{\mu}$-attracting neighborhood of $A_{\mu}$.

Proof. $\phi(U \times \Lambda(U))$ is an $X$-neighborhood of $A_{\lambda}$, and therefore there exists $Y_{0}$, a $\Gamma$-neighborhood of $A_{\lambda}$, with $Y_{0} \cap X=\phi(U \times \Lambda(U)) . Y_{0} \cap S_{\lambda}$ is an attracting neighborhood of $A_{\lambda}$. By Proposition 2.1, there exists a $\Gamma$-open neighborhood $Y_{1}$ of $S_{\lambda}$ such that if $\widehat{S} \subset Y_{1}$ is compact invariant, then $Y_{0} \cap \widehat{S}$ is an attracting neighborhood of some attractor in $\widehat{S}$. Let $V_{0} \subset Z$ be such that $\phi_{\lambda}\left(V_{0}\right)$ is an $X_{\lambda^{-}}$ isolating neighborhood of $S_{\lambda}$ contained in $\phi_{\lambda}(V) \cap Y_{1}$, and set $U_{0}=V_{0} \cap U$. There exists an open neighborhood $W_{0}$ of $\lambda$ such that $\phi\left(V_{0} \times W_{0}\right) \subset Y_{1}, \sigma_{V_{0}}(\lambda)=\sigma_{V}(\lambda)=$ $S_{\lambda}$. By Proposition 4.3, there exists $W_{1}$, an open neighborhood of $\lambda$ contained in $\Lambda\left(V_{0}\right) \cap \Lambda(V)$, such that $\mu \in W_{1}$ implies $\sigma_{V_{0}}(\mu)$ and $\sigma_{V}(\mu)$ have the same maximal invariant set. Similarly, there exists $W_{2}$, an open neighborhood of $\lambda$ contained in $\Lambda\left(U_{0}\right) \cap \Lambda(U)$, such that $\mu \in W_{2}$ implies $\sigma_{U_{0}}(\mu)$ and $\sigma_{U}(\mu)$ have the same maximal invariant set. Set $W=W_{0} \cap W_{1} \cap W_{2}$.

If $\mu \in W$, then $\sigma_{V}(\mu)=\sigma_{V_{0}}(\mu)=S_{\mu}$ and $\sigma_{U}(\mu)=\sigma_{U_{0}}(\mu)=A_{\mu}$ for some invariant sets $S_{\mu}$ and $A_{\mu}$ in $X_{\mu}$. We claim that $A_{\mu}$ is an attractor in $S_{\mu}$ with $X_{\mu}$-isolating $S_{\mu}$-attracting neighborhood $\phi_{\mu}(U) . \mu \in W \subset W_{0}$ implies $S_{\mu} \subset Y_{1}$. Therefore, $Y_{0} \cap S_{\mu}\left(=\phi_{\mu}(U) \cap S_{\mu}\right)$ is an attracting neighborhood of an attractor $A_{\mu}^{\prime}$ in $S_{\mu}$. Since $A_{\mu}$ is the maximal invariant set in $\phi_{\mu}(U)$, it follows that $A_{\mu}^{\prime}=A_{\mu} \cap S_{\mu}$. However, $A_{\mu}$ is the maximal invariant set in $\phi_{\mu}\left(U_{0}\right)$, and $\phi_{\mu}\left(U_{0}\right) \subset \phi_{\mu}\left(V_{0}\right)$ implies $A_{\mu} \subset S_{\mu}$. Therefore $A_{\mu}^{\prime}=A_{\mu}$, yielding the claim.

Assume $U, V, W$ are as in Proposition 4.6, and for $\mu \in W$, let $A_{\mu}=\sigma_{U}(\mu)$ and $S_{\mu}=\sigma_{V}(\mu) . A_{\mu}$ is an attractor in $S_{\mu}$; let $A_{\mu}^{*}$ denote the complementary repeller. The following proposition implies that $A_{\mu}^{*}$ is the continuation of $A_{\lambda}^{*}$. 
PROPOSITION 4.7. $W \subset \Lambda\left(\operatorname{cl}_{Z}(V \backslash U)\right)$, and for each $\mu \in W, \phi_{\mu}\left(\operatorname{cl}_{Z}(V \backslash U)\right)$ is an $X_{\mu}$-isolating neighborhood of $A_{\mu}^{*}$.

PROOF. $\phi_{\mu}(V)$ is an $X_{\mu}$-isolating neighborhood of $S_{\mu}$, and $\phi_{\mu}(U)$ is an $X_{\mu^{-}}$ isolating $S_{\mu}$-attracting neighborhood of $A_{\mu}$. Proposition 3.1 implies that

$$
\operatorname{cl}_{X_{\mu}}\left(\phi_{\mu}(V) \backslash \phi_{\mu}(U)\right) \quad\left(=\phi_{\mu}\left(\operatorname{cl}_{Z}(V \backslash U)\right)\right)
$$

is an $X_{\mu}$-isolating neighborhood of $A_{\mu}^{*}$, and therefore $\mu \in \Lambda\left(\mathrm{cl}_{Z}(V \backslash U)\right)$.

By Proposition 4.5, if $\left(A_{\lambda}, A_{\lambda}^{*}\right)$ is an attractor-repeller pair in the isolated invariant set $S_{\lambda}$, then for $\mu$ near $\lambda$, there are isolated invariant sets $A_{\mu}, A_{\mu}^{*}$, and $S_{\mu}$ near $A_{\lambda}, A_{\lambda}^{*}$, and $S_{\lambda}$, respectively. Propositions 4.6 and 4.7 then imply that $\left(A_{\mu}, A_{\mu}^{*}\right)$ is an attractor-repeller pair in $S_{\mu}$. This is made more formal in

THEOREM 4.8. The maps $\pi_{\mathscr{A} A}, \mathscr{P}_{A}, \mathscr{P}_{A^{*}}$, and $\mathscr{P}_{S}$ are surjective local homeomorphisms.

ProOF. Assume $\left(A_{\lambda}, A_{\lambda}^{*}, S_{\lambda}\right) \in \mathscr{A} \mathscr{R}$. Choose $V$, an $X_{\lambda}$-isolating neighborhood of $S_{\lambda}$, and $U$, an $X_{\lambda}$-isolating $S_{\lambda}$-attracting neighborhood of $A_{\lambda}$. Set $U^{\prime}=$ $\operatorname{cl}_{Z}(V \backslash U)$.

Let $W$ be as in Proposition 4.6. $W \subset \Lambda(U) \cap \Lambda\left(U^{\prime}\right) \cap \Lambda(V)$ by Propositions 4.6 and 4.7. Define $\tau: W \rightarrow \mathscr{S} \times \mathscr{S} \times \mathscr{S}, \tau=\sigma_{U}\left|W \times \sigma_{U^{\prime}}\right| W \times \sigma_{V} \mid W$. Clearly, $\tau$ is continuous. The above results imply $\operatorname{im}(\tau) \subset \mathscr{A} \mathscr{R}$. Therefore, $\tau$ defines a continuous map $\tau: W \rightarrow \mathscr{A} \mathscr{R}$. Since $\pi_{\mathscr{A} \mathscr{A}}$ is continuous, so is $\pi_{\mathscr{R} R} \mid \operatorname{im}(\tau): \operatorname{im}(\tau) \rightarrow$ $W$. It is not difficult to see that $\tau: W \rightarrow \operatorname{im}(\tau)$ and $\pi_{\mathscr{Q R} R} \mid \operatorname{im}(\tau): \operatorname{im}(\tau) \rightarrow W$ are inverse bijections and therefore inverse homeomorphisms. To prove that $\pi_{\mathscr{A}:}$ is a local homeomorphism, we need to show that $\operatorname{im}(\tau)$ is an open subset of $\mathscr{A} \mathscr{R}$.

Let $Y=\sigma_{U}(W) \times \sigma_{U^{\prime}}(W) \times \sigma_{V}(W) . Y$ is an open subset of $\mathscr{S} \times \mathscr{S} \times \mathscr{S}$; therefore, $Y \cap \mathscr{A R}$ is an open subset of $\mathscr{A} \mathscr{R}$. Clearly, $Y \cap \mathscr{A} \mathscr{R} \subset \operatorname{im}(\tau)$. By the definition of $\tau, \operatorname{im}(\tau) \subset Y$. Since $\operatorname{im}(\tau) \subset \mathscr{A} \mathscr{R}$, it then follows that $\operatorname{im}(\tau)=Y \cap \mathscr{A} \mathscr{R} ;$ i.e., $\operatorname{im}(\tau)$ is an open subset of $\mathscr{A} \mathscr{R}$. Thus $\pi_{\mathscr{A} \mathscr{A}}$ is a local homeomorphism. $\pi_{\mathscr{A}} \mathscr{R}$ is surjective because if $\lambda \in \Lambda$ and $A_{\lambda}=A_{\lambda}^{*}=S_{\lambda}=\varnothing$, then $\left(A_{\lambda}, A_{\lambda}^{*}\right)$ is an attractor-repeller pair in the $X_{\lambda}$-isolated invariant set $S_{\lambda}$ and $\pi_{\mathscr{0} / \mathscr{A}}\left(A_{\lambda}, A_{\lambda}^{*}, S_{\lambda}\right)=\lambda$.

To see that $\mathscr{P}_{S}$ is a local homeomorphism, first note that $\mathscr{P}_{S}(\operatorname{im}(\tau))=\sigma_{V}(W)$. Now consider

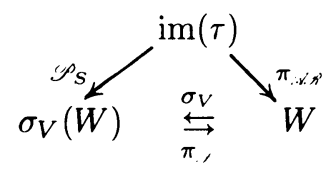

This diagram commutes, and the maps on the base and right side are homeomorphisms. Therefore, $\mathscr{P}_{S} \mid \operatorname{im}(\tau): \operatorname{im}(\tau) \rightarrow \sigma_{V}(W)$ is a homeomorphism, implying that $\mathscr{P}_{S}$ is a local homeomorphism. $\mathscr{P}_{S}$ is surjective because if $S_{\lambda} \in \mathscr{S}$, then $\left(S_{\lambda}, \phi\right)$ is an attractor-repeller pair in the $X_{\lambda}$-isolated invariant set $S_{\lambda}$ and $\mathscr{P}_{S}\left(S_{\lambda}, \phi, S_{\lambda}\right)=S_{\lambda}$.

The proof that $\mathscr{P}_{A}$ and $\mathscr{P}_{A^{*}}$ are surjective local homeomorphisms is similar.

We say that the attractor-repeller pairs $\left(A_{\lambda}, A_{\lambda}^{*}\right)$ in $S_{\lambda} \subset X_{\lambda}$ and $\left(A_{\mu}, A_{\mu}^{*}\right)$ in $S_{\mu} \subset X_{\mu}$ are related by continuation or are continuations of each other if there exists a path $c$ in $\mathscr{A} \mathscr{R}$ from $\left(A_{\lambda}, A_{\lambda}^{*}, S_{\lambda}\right)$ to $\left(A_{\mu}, A_{\mu}^{*}, S_{\mu}\right)$. Under this circumstance, $\mathscr{P}_{A} c, \mathscr{P}_{A^{*}} c$, and $\mathscr{P}_{S} c$ are paths in $\mathscr{S}$ from $A_{\lambda}$ to $A_{\mu}$, from $A_{\lambda}^{*}$ to $A_{\mu}^{*}$, and from $S_{\lambda}$ 
to $S_{\mu}$, respectively; i.e., $A_{\lambda}$ and $A_{\mu}, A_{\lambda}^{*}$ and $A_{\mu}^{*}$, and $S_{\lambda}$ and $S_{\mu}$ are, in each case, related by continuation.

Next, we extend these results to obtain the continuation theory for (<-ordered) Morse decompositions.

DEFINITION 4.9. The space of Morse decompositions (indexed by $P$ ) for the product parameterization $\phi$ is the set

$$
\mathscr{M}_{P}=\mathscr{M}_{P}(\phi)=\left\{\underset{\pi \in P}{\times} M_{\lambda}(\pi) \times S_{\lambda} \in \underset{P}{\times} \mathscr{S}(\phi) \times \mathscr{S}(\phi) \mid \begin{array}{l}
\left\{M_{\lambda}(\pi)\right\}_{\pi \in P} \text { is a Morse } \\
\text { decomposition of } S_{\lambda}
\end{array}\right\}
$$

with the topology inherited as a subspace of the product space $\times_{P} \mathscr{S}(\phi) \times \mathscr{S}(\phi)$.

Note that the $S_{\lambda}$ component is needed in the definition of $\mathscr{M}_{P}$ because, otherwise, if a collection of invariant sets is a Morse decomposition of more than one invariant set, then there is no distinction between the Morse decompositions. Of course, it is precisely for this reason that the structures related to a Morse decomposition of an invariant set $S$ are defined relative to $S$. In particular, without the $S_{\lambda}$ component, the homology index braid and connection matrices are not well defined on $\mathscr{M}_{P}$ and the continuation theories for these indices cannot be stated.

Recall that $<$ is a partial order on $P$.

DEFINITION 4.10. The space of <-ordered Morse decompositions for the product parameterization $\phi$ is the set

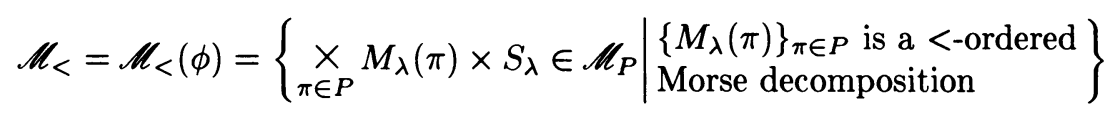

with the topology inherited as a subspace of $\mathscr{M}_{P}$.

Define $\pi_{\mathscr{M}_{P}}: \mathscr{M}_{P} \rightarrow \Lambda$, where $\pi_{\mathscr{M}_{P}}\left(\times_{\pi \in P} M_{\lambda}(\pi) \times S_{\lambda}\right)=\lambda$, and $\pi_{\mathscr{K}_{<}}: \mathscr{M}_{<} \rightarrow \Lambda$, where $\pi_{\mathscr{M}_{<}}=\pi_{\mathscr{M}_{P}} \mid \mathscr{M}_{<}$.

If $M=\times_{\pi \in P} M_{\lambda}(\pi) \times S_{\lambda} \in \mathscr{M}_{<}$and $I \in I(<)$, then

$$
M_{\lambda}(I)=\left(\bigcup_{\pi \in I} M_{\lambda}(\pi)\right) \cup\left(\bigcup_{\pi, \pi^{\prime} \in I} C\left(M_{\lambda}(\pi), M_{\lambda}\left(\pi^{\prime}\right)\right)\right)
$$

is an $X_{\lambda}$-isolated invariant set. For each $I \in I(<)$, define $\mathscr{P}_{I}: \mathscr{M}_{<} \rightarrow \mathscr{S}$ to be the map that sends $M$ to $M_{\lambda}(I)$. If $(I, J) \in I_{2}(<)$, then $\left(M_{\lambda}(I), M_{\lambda}(J)\right)$ is an attractorrepeller pair in $M_{\lambda}(I J)$. For each $(I, J) \in I_{2}(<)$, define $\mathscr{P}_{I, J}: \mathscr{M}_{<} \rightarrow \mathscr{A} \mathscr{R}$ to be the map that sends $M$ to $\left(M_{\lambda}(I), M_{\lambda}(J), M_{\lambda}(I J)\right)$.

For each $(I, J) \in I_{2}(<)$, we have the following commutative diagrams of maps.

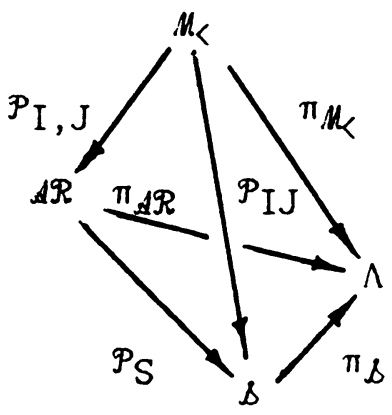



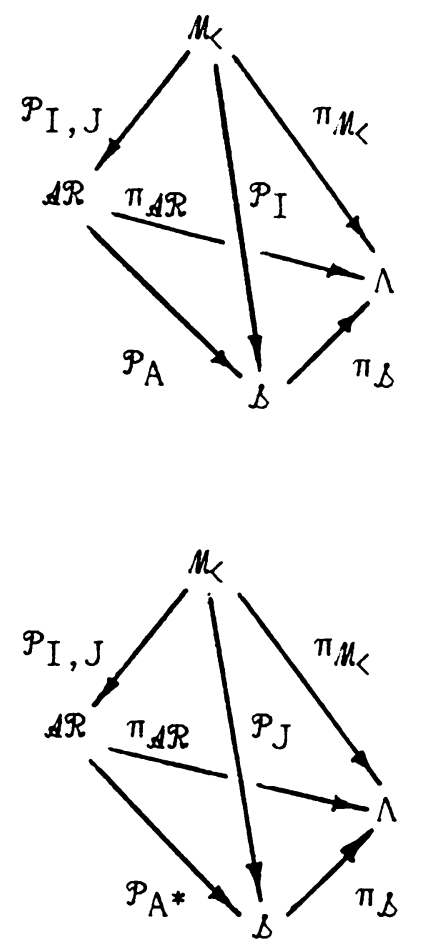

Consider each diagram as a tetrahedron of maps. By above, the maps on the base are surjective local homeomorphisms. After a couple of preliminary propositions, we prove that $\pi_{\mathscr{U}_{P}}, \pi_{\mathscr{K}_{<}}, \mathscr{P}_{I}$ (and therefore $\mathscr{P}_{J}$ and $\mathscr{P}_{I J}$ ), and $\mathscr{P}_{I, J}$ are surjective local homeomorphisms.

The idea is as follows. If $M_{\lambda}$ is a <-ordered Morse decomposition of the isolated invariant set $S_{\lambda} \subset X_{\lambda}$ and $A F(<)$ is the associated attractor filtration, then for $\mu$ near $\lambda$, Proposition 4.6 implies that the attractors contained in $A F(<)$ continue to attractors in $S_{\mu}$, the continuation of $S_{\lambda}$. In Proposition 4.11, we prove that $A F_{\mu}$, the collection of continued attractors in $S_{\mu}$, is a <-consistent attractor filtration. Then, in Proposition 4.12, we prove that if $M_{\mu}$ is the <-ordered Morse decomposition defined by $A F_{\mu}$, then for each $I \in I(<)$, the Morse set $M_{\mu}(I)$ is the continuation of the Morse set $M_{\lambda}(I)$, and therefore the <-ordered Morse decomposition $M_{\lambda}$ continues to the <-ordered Morse decomposition $M_{\mu}$.

Proposition 4.11. Assume $M_{\lambda}=\left\{M_{\lambda}(\pi)\right\}_{\pi \in P}$ is a <-ordered Morse decomposition of an isolated invariant set $S_{\lambda} \subset X_{\lambda}$. Let $\left\{M_{\lambda}(I) \mid I \in A(<)\right\}$ be the attractor filtration of the associated admissible ordering of $M_{\lambda}$, and for each $I \in A(<)$, assume $U_{I} \subset_{-} Z$ is such that $\phi_{\lambda}\left(U_{I}\right)$ is an $X_{\lambda}$-isolating $S_{\lambda}$-attracting neighborhood of $M_{\lambda}(I)$. Further, assume $U_{\phi}=\phi$ and $U_{I} \subset U_{P}$ for each $I \in A(<)$. Then there exists $W$, an open neighborhood of $\lambda$ contained in each $\Lambda\left(U_{I}\right)$, such that $\mu \in W$ implies

(1) if $\sigma_{U_{I}}(\mu)=M_{\mu}(I)$, then the collection $A F_{\mu}:=\left\{M_{\mu}(I) \mid I \in A(<)\right\}$ is a $<-$ consistent attractor filtration in $S_{\mu}:=M_{\mu}(P)$, 
(2) for each $I \in A(<), \phi_{\mu}\left(U_{I}\right)$ is an $X_{\mu}$-isolating $S_{\mu}$-attracting neighborhood of $M_{\mu}(I)$.

ProOF. If $I, I^{\prime} \in A(<)$, then $\phi_{\lambda}\left(U_{I \cup I^{\prime}}\right)$ and $\phi_{\lambda}\left(U_{I}\right) \cup \phi_{\lambda}\left(U_{I^{\prime}}\right)\left(=\phi_{\lambda}\left(U_{I} \cup U_{I^{\prime}}\right)\right)$ are both isolating neighborhoods (the latter by Proposition 3.2) of the invariant set $M_{\mu}\left(I \cup I^{\prime}\right)$. Proposition 4.3 implies that there exists an open neighborhood $W_{I, I^{\prime}}$ of $\lambda$ such that if $\mu \in W_{I, I^{\prime}}$, then $\phi_{\mu}\left(U_{I \cup I^{\prime}}\right)$ and $\phi_{\mu}\left(U_{I} \cup U_{I^{\prime}}\right)$ are isolating neighborhoods of the same $X_{\mu}$-isolated invariant set. Similarly, there exists an open neighborhood $V_{I, I^{\prime}}$ of $\lambda$ such that $\mu \in V_{I, I^{\prime}}$ implies that $\phi_{\mu}\left(U_{I \cap I^{\prime}}\right)$ and $\phi_{\mu}\left(U_{I} \cap U_{I^{\prime}}\right)$ are isolating neighborhoods of the same $X_{\mu}$-isolated invariant set. Given $I \in A(<)$, Proposition 4.6 implies that there exists $W_{I}$, an open neighborhood of $\lambda$ contained in $\Lambda\left(U_{I}\right) \cap \Lambda\left(U_{P}\right)$, such that $\mu \in W_{I}$ implies that if $M_{\mu}(I)=\sigma_{U_{I}}(\mu)$ and $S_{\mu}=\sigma_{U_{P}}(\mu)$, then $M_{\mu}(I)$ is an attractor in $S_{\mu}$ and $\phi_{\mu}\left(U_{I}\right)$ is an $X_{\mu}$-isolating $S_{\mu}$-attracting neighborhood of $M_{\mu}(I)$.

Let $W$ be an open neighborhood of $\lambda$ contained in each $W_{I, I^{\prime}}$, each $V_{I, I^{\prime}}$, and each $W_{I}$.

Assume $\mu \in W$. Define $M_{\mu}(I)=\sigma_{U_{I}}(\mu)$ for $I \in A(<)$ and $S_{\mu}=\sigma_{U_{P}}(\mu)$. Then $M_{\mu}(I)$ is an attractor in $S_{\mu}$ and $\phi_{\mu}\left(U_{I}\right)$ is an $X_{\mu}$-isolating $S_{\mu}$-attracting neighborhood of $M_{\mu}(I)$. Set $A F_{\mu}=\left\{M_{\mu}(I) \mid I \in A(<)\right\}$. We claim that $A F_{\mu}$ is a <-consistent attractor filtration in $S_{\mu}$. First, note that since $U_{\phi}=\varnothing$, it follows that $M_{\mu}(\phi)=\varnothing$, and therefore $\phi \in A F_{\mu}$. Clearly, $S_{\mu} \in A F_{\mu}$. We need to show that $I, I^{\prime} \in A(<)$ implies $M_{\mu}\left(I \cup I^{\prime}\right)=M_{\mu}(I) \cup M_{\mu}\left(I^{\prime}\right)$ and $M_{\mu}\left(I \cap I^{\prime}\right)=$ $M_{\mu}(I) \cap M_{\mu}\left(I^{\prime}\right) . \quad M_{\mu}(I), M_{\mu}\left(I^{\prime}\right)$, and $M_{\mu}\left(I \cup I^{\prime}\right)$ are attractors in $S_{\mu}$ with $X_{\mu}$ isolating $S_{\mu}$-attracting neighborhoods $\phi_{\mu}\left(U_{I}\right), \phi_{\mu}\left(U_{I^{\prime}}\right)$, and $\phi_{\mu}\left(U_{I \cup I^{\prime}}\right)$, respectively. Proposition 3.2 implies that $\phi_{\mu}\left(U_{I}\right) \cup \phi_{\mu}\left(U_{I^{\prime}}\right)\left(=\phi_{\mu}\left(U_{I} \cup U_{I^{\prime}}\right)\right)$ is an $X_{\mu}$-isolating $S_{\mu^{-}}$ attracting neighborhood of the attractor $M_{\mu}(I) \cup M_{\mu}\left(I^{\prime}\right) . W \subset W_{I, I^{\prime}} ;$ therefore, the maximal invariant set in $\phi_{\mu}\left(U_{I} \cup U_{I^{\prime}}\right)$ is equal to that in $\phi_{\mu}\left(U_{I \cup I^{\prime}}\right)$. Thus, $M_{\mu}(I) \cup M_{\mu}\left(I^{\prime}\right)=M_{\mu}\left(I \cup I^{\prime}\right)$. Similarly, $M_{\mu}(I) \cap M_{\mu}\left(I^{\prime}\right)=M_{\mu}\left(I \cap I^{\prime}\right)$, and therefore $A F_{\mu}$ is a <-consistent attractor filtration in $S_{\mu}$.

Assume $U_{I}$ and $W$ are as described in Proposition 4.11, and for $\mu \in W$, let $A F_{\mu}$ be the <-consistent attractor filtration in $S_{\mu}$ defined above. Assume $M_{\mu}=$ $\left\{M_{\mu}(\pi)\right\}_{\pi \in P}$ is the <-ordered Morse decomposition of $S_{\mu}$ defined by $A F_{\mu}$, and let $\left\{M_{\mu}(J) \mid J \in I(<)\right\}$ be the Morse sets of the associated admissible ordering of $M_{\mu}$. The following proposition is a direct consequence of Propositions 3.3 and 4.11 and implies that the Morse set $M_{\mu}(J)$ is the continuation of the Morse set $M_{\lambda}(J)$ for each $J \in I(<)$.

Proposition 4.12. If $J \in I(<)$ and $(I, J)$ is a decomposition of an interval $K \in A(<)$, then $W \subset \Lambda\left(\operatorname{cl}_{Z}\left(U_{K} \backslash U_{I}\right)\right)$ and $\phi_{\mu}\left(\operatorname{cl}_{Z}\left(U_{K} \backslash U_{I}\right)\right)$ is an $X_{\mu}$-isolating neighborhood of $M_{\mu}(J)$.

Thus, Propositions 4.11 and 4.12 imply that if $\left\{M_{\lambda}(\pi)\right\}_{\pi \in P}$ is a <-ordered Morse decomposition of $S_{\lambda}$, then for $\mu$ near $\lambda$, there are isolated invariant sets $M_{\mu}(\pi)$ near $M_{\lambda}(\pi)$ and $S_{\mu}$ near $S_{\lambda}$ such that $\left\{M_{\mu}(\pi)\right\}_{\pi \in P}$ is <-ordered Morse decomposition of $S_{\mu}$. Therefore Morse decompositions and admissible orderings continue locally. It is noteworthy that while the flow ordering of a Morse decomposition continues locally to an admissible ordering of nearby Morse decompositions, it does not necessarily continue to the flow ordering. This is illustrated in an example below and plays 
an important role in the connection matrix continuation theory. We make the above-described continuation relationships more formal with the following

THEOREM 4.13. The maps $\mathscr{P}_{I}$ for $I \in I(<), \mathscr{P}_{I, J}$ for $(I, J) \in I_{2}(<), \pi_{\mathscr{M}_{P}}$, and $\pi_{\mathscr{H}_{<}}$are surjective local homeomorphisms.

Proof. Assume $M=\times_{\pi \in P} M_{\lambda}(\pi) \times S_{\lambda} \in \mathscr{M}_{P} . \quad\left\{M_{\lambda}(\pi)\right\}_{\pi \in P}$ is a Morse decomposition of $S_{\lambda}$. Let $<$ be an admissible ordering of the Morse decomposition. $\left\{M_{\lambda}(I) \mid I \in A(<)\right\}$ is the associated attractor filtration, and $\left\{M_{\lambda}(I) \mid I \in I(<)\right\}$ is the associated collection of Morse sets.

Set $U_{\phi}=\varnothing$, and for each nonempty $I \in A(<)$, let $U_{I} \subset Z$ be such that $\phi_{\lambda}\left(U_{I}\right)$ is an $X_{\lambda}$-isolating $S_{\lambda}$-attracting neighborhood of $M_{\lambda}(I)$ contained in $\phi_{\lambda}\left(U_{P}\right)$. Given $J \in I(<) \backslash A(<)$, choose $I, K \in A(<)$ such that $(I, J)$ is a decomposition of $K$, and set $U_{J}=\operatorname{cl}_{Z}\left(U_{K} \backslash U_{I}\right)$. Let $W$ be as in Proposition 4.11. By Propositions 4.11 and 4.12, $W \subset \Lambda\left(U_{J}\right)$ for each $J \in I(<)$. For $\mu \in W$ and $J \in I(<)$, set $M_{\mu}(J)$ equal to the maximal invariant set in $\phi_{\mu}\left(U_{J}\right)$. Propositions 4.11 and $4.12 \mathrm{imply}$ that $\left\{M_{\mu}(\pi) \mid \pi \in P\right\}$ is a <-ordered Morse decomposition of $S_{\mu}:=M_{\mu}(P)$ and $M S_{\mu}:=\left\{M_{\mu}(J) \mid J \in I(<)\right\}$ is the associated collection of Morse sets.

Define $\tau: W \rightarrow \times_{\pi \in P} \mathscr{S} \times \mathscr{S}, \tau=\times_{\pi \in P} \sigma_{U_{\pi}}\left|W \times \sigma_{U_{P}}\right| W$. Clearly, $\tau$ is continuous. The above results imply $\tau(\mu)=\times_{\pi \in P} M_{\mu}(\pi) \times S_{\mu}$, and therefore $M \in \operatorname{im}(\tau) \subset \mathscr{M}_{<} \subset \mathscr{M}_{P}$. Thus, $\tau$ defines a continuous map, $\tau: W \rightarrow \mathscr{M}_{P}$. Since $\pi_{\mathscr{M}_{P}}$ is continuous, so is $\pi_{\mathscr{M}_{P}} \mid \operatorname{im}(\tau): \operatorname{im}(\tau) \rightarrow W$. It is not difficult to see that $\pi_{\mathscr{M}_{P}} \mid \operatorname{im}(\tau): \operatorname{im}(\tau) \rightarrow W$ and $\tau: W \rightarrow \operatorname{im}(\tau)$ are inverse bijections and therefore inverse homeomorphisms. To complete the proof that $\pi_{\mathscr{K}_{P}}$ is a local homeomorphism, we need to show that $\operatorname{im}(\tau)$ is an open subset of $\mathscr{M}_{P}$.

Let $V=\times_{\pi \in P} \sigma_{U_{\pi}}(W) \times \sigma_{U_{P}}(W) . V$ is open in $\times_{P} \mathscr{S} \times \mathscr{S}$; therefore, $V \cap \mathscr{M}_{P}$ is open in $\mathscr{M}_{P}$. Clearly, $V \cap \mathscr{M}_{P} \subset \operatorname{im}(\tau)$. By the definition of $\tau, \operatorname{im}(\tau) \subset V$. Since $\operatorname{im}(\tau) \subset \mathscr{M}_{P}$, it now follows that $\operatorname{im}(\tau)=V \cap \mathscr{M}_{P}$; i.e., $\operatorname{im}(\tau)$ is an open subset of $\mathscr{M}_{P}$. Thus, $\pi_{\mathscr{M}_{P}}$ is a local homeomorphism.

Since $\operatorname{im}(\tau) \subset \mathscr{M}_{<} \subset \mathscr{M}_{P}$ and $\pi_{\mathscr{K}_{<}}=\pi_{\mathscr{M}_{P}} \mid \mathscr{M}_{<}$, it follows that $\pi_{\mathscr{K}_{<}}$is a local homeomorphism.

$\pi_{\mathscr{K}_{P}}$ is surjective because if $\lambda \in \Lambda$ and $M_{\lambda}(\pi)=\varnothing$ for each $\pi \in P$, then $\left\{M_{\lambda}(\pi)\right\}_{\pi \in P}$ is a Morse decomposition of the $X_{\lambda}$-isolated invariant set $S_{\lambda}=\phi$ and $\pi_{\mathscr{H}_{P}}\left(\times_{\pi \in P} M_{\lambda}(\pi) \times S_{\lambda}\right)=\lambda$. Similarly, $\pi_{\mathscr{K}_{<}}$is surjective.

Assume $I \in I(<)$. To see that $\mathscr{P}_{I}$ is a local homeomorphism, first note that $\mathscr{P}_{I}(\operatorname{im}(\tau))=\sigma_{U_{I}}(W)$. Now consider the following diagram:



The diagram commutes, and the maps on the base and the right side are homeomorphisms. Therefore, $\mathscr{P}_{I} \mid \operatorname{im}(\tau): \operatorname{im}(\tau) \rightarrow \sigma_{U_{I}}(W)$ is a homeomorphism, implying that $\mathscr{P}_{I}$ is a local homeomorphism.

To see that $\mathscr{P}_{I}$ is surjective, assume $S_{\lambda} \in \mathscr{P}$ and choose $\pi^{\prime} \in I$. Set $M_{\lambda}\left(\pi^{\prime}\right)=S_{\lambda}$ and $M_{\lambda}(\pi)=\varnothing$ for $\pi \in P$ with $\pi \neq \pi^{\prime} . \quad\left\{M_{\lambda}(\pi)\right\}_{\pi \in P}$ is a <-ordered Morse decomposition of the $X_{\lambda}$-isolated invariant set $S_{\lambda}$ and $\mathscr{P}_{I}\left(\times_{\pi \in P} M_{\lambda}(\pi) \times S_{\lambda}\right)=S_{\lambda}$.

For $(I, J) \in I_{2}(<)$, it similarly follows that $\mathscr{P}_{I, J}$ is a surjective local homeomorphism. 
The proof of the following important property of $\mathscr{M}_{<}$is contained in the proof of Theorem 4.13 .

Proposition 4.14. $\mathscr{M}_{<}$is an open subset of $\mathscr{M}_{P}$.

DEFINITION 4.15. Let $M_{\lambda}=\left\{M_{\lambda}(\pi)\right\}_{\pi \in P}$ and $M_{\mu}=\left\{M_{\mu}(\pi)\right\}_{\pi \in P}$ be Morse decompositions of isolated invariant sets $S_{\lambda} \subset X_{\lambda}$ and $S_{\mu} \subset X_{\mu}$, respectively. We say that $M_{\lambda}$ and $M_{\mu}$ are related by continuation or are continuations of each other if there exists a path $c$ in $\mathscr{M}_{P}$ from $\times_{\pi \in P} M_{\lambda}(\pi) \times S_{\lambda}$ to $\times_{\pi \in P} M_{\mu}(\pi) \times S_{\mu}$. If, furthermore, $M_{\lambda}$ and $M_{\mu}$ are <-ordered and the path $c$ is in $\mathscr{M}_{<}$, then we say that the associated admissible orderings are related by continuation or are continuations of each other.

PROPOSITION 4.16. Let $M_{\lambda}=\left\{M_{\lambda}(\pi)\right\}_{\pi \in P}$ and $M_{\mu}=\left\{M_{\mu}(\pi)\right\}_{\pi \in P}$ be <ordered Morse decompositions, and assume that the associated admissible orderings are related by continuation. Then the Morse sets $M_{\lambda}(I)$ and $M_{\mu}(I)$ are related by continuation for each $I \in I(<)$, and the attractor-repeller pairs $\left(M_{\lambda}(I), M_{\lambda}(J)\right)$ in $M_{\lambda}(I J)$ and $\left(M_{\mu}(I), M_{\mu}(J)\right)$ in $M_{\mu}(I J)$ are related by continuation for each $(I, J) \in I_{2}(<)$.

PROOF. If $c$ is a path in $\mathscr{M}_{<}$from $\times_{\pi \in P} M_{\lambda}(\pi) \times S_{\lambda}$ to $\times_{\pi \in P} M_{\mu}(\pi) \times S_{\mu}$, then $\mathscr{P}_{I} c$ is a path in $\mathscr{S}$ from $M_{\lambda}(I)$ to $M_{\mu}(I)$, and $\mathscr{P}_{I, J} c$ is a path in $\mathscr{A} \mathscr{R}$ from $\left(M_{\lambda}(I), M_{\lambda}(J), M_{\lambda}(I J)\right)$ to $\left(M_{\mu}(I), M_{\mu}(J), M_{\mu}(I J)\right)$.

We illustrate the spaces of ordered Morse decompositions with the following simple example.

Consider the following family of local flows which qualitatively represent the solutions to the family of differential equations, $\dot{x}=y, \dot{y}=\theta y-x\left(x-\frac{1}{3}\right)(1-x)$, for $\theta>0$ (see $\S 6)$.

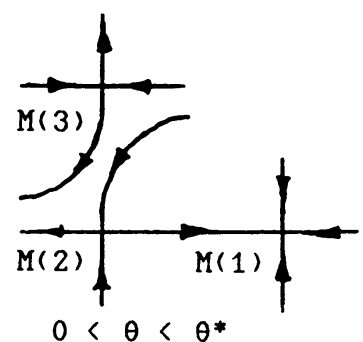

$0<\theta<\theta^{*}$

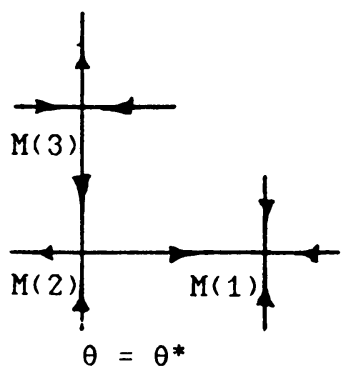

$\theta=\theta^{*}$

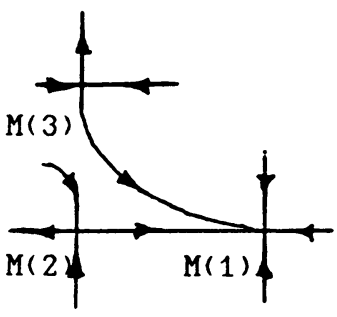

$\theta>\theta^{*}$

$$
\left.\Lambda=\stackrel{0}{\left(-\theta^{*}\right.}\right)^{\infty}
$$

Let $P=\{1,2,3\}$, and for each $\theta>0$, let $M_{\theta}:=\{M(\pi)\}_{\pi \in P}$ be the Morse decomposition pictured. $\mathscr{M}_{P}$ contains 
Thus, for $\theta$ near 0 and for $\theta$ large, the Morse decompositions $M_{\theta}$ are related by continuation. Let $<$ be the usual total order on $P$. $\mathscr{M}_{<}$contains

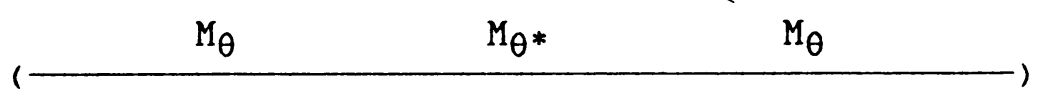

Thus, the admissible ordering $<$ of $M_{\theta}$ for $\theta$ near 0 is related by continuation to the admissible ordering $<$ of $M_{\theta}$ for $\theta$ large. Let $<^{\prime}$ be the partial order on $P$ with relations $1<^{\prime} 2,1<^{\prime} 3$. $\mathscr{M}_{<^{\prime}}$ contains

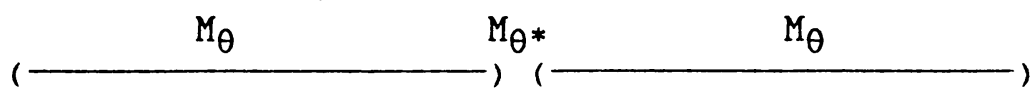

Therefore, the admissible ordering $<^{\prime}$ of $M_{\theta}$ for $\theta$ near 0 does not continue to the admissible ordering $<^{\prime}$ of $M_{\theta}$ for $\theta$ large.

This example illustrates the above-proven fact that Morse decompositions and admissible orderings continue locally. It also establishes the fact that although the flow ordering continues locally to an admissible ordering, it need not continue to the flow ordering. This follows because $<$ is the flow ordering of $M_{\theta^{*}}$ while $<^{\prime}$ is the flow ordering of $M_{\theta}$ for $\theta>\theta^{*}$.

5. The continuation theory for homology index braids and connection matrices. Assume $\phi: Z \times \Lambda \rightarrow X$ is a product parameterization of the local flow $X$, and let $\mathscr{S}, \mathscr{A} \mathscr{R}, \mathscr{M}_{P}$, and $\mathscr{M}_{<}$be the spaces of invariant sets, attractor-repeller pairs, Morse decompositions indexed by $P$, and <-ordered Morse decompositions, respectively, for the product parameterization.

The Conley index is invariant under continuation by

THEOREM 5.1 (SEE [1]). If $S_{\lambda}$ and $S_{\mu}$ are related by continuation and $c$ is a path in $\mathscr{S}$ from $S_{\lambda}$ to $S_{\mu}$, then (associated to the path-homotopy class of $c$ ) there is a homotopy equivalence $\theta_{[c]}: h\left(S_{\lambda}\right) \rightarrow h\left(S_{\mu}\right)$.

Our use of Theorem 5.1 is on the homology level; thus,

COROLlaRY 5.2. If $c$ is as in Theorem 5.1, then (associated to the pathhomotopy class of $c$ ) there is an isomorphism $\theta_{[c]}: H\left(S_{\lambda}\right) \rightarrow H\left(S_{\mu}\right)$.

If the attractor-repeller pairs $\left(A_{\lambda}, A_{\lambda}^{*}\right)$ in $S_{\lambda}$ and $\left(A_{\mu}, A_{\mu}^{*}\right)$ in $S_{\mu}$ are related by continuation and $c$ is a path in $\mathscr{A} \mathscr{R}$ from $\left(A_{\lambda}, A_{\lambda}^{*}, S_{\lambda}\right)$ to $\left(A_{\mu}, A_{\mu}^{*}, S_{\mu}\right)$, then $\mathscr{P}_{A} c$, $\mathscr{P}_{A^{*}} c$, and $\mathscr{P}_{S} c$ are paths in $\mathscr{S}$ from $A_{\lambda}$ to $A_{\mu}$, from $A_{\lambda}^{*}$ to $A_{\mu}^{*}$, and from $S_{\lambda}$ to $S_{\mu}$, respectively. Therefore, there are isomorphisms $\theta_{\left[\mathscr{P}_{A} c\right]}: H\left(A_{\lambda}\right) \rightarrow H\left(A_{\mu}\right)$, $\theta_{\left[\mathscr{P}_{\left.A^{*} c\right]}\right.}: H\left(A_{\lambda}^{*}\right) \rightarrow H\left(A_{\mu}^{*}\right)$, and $\theta_{\left[\mathscr{P}_{S} c\right]}: H\left(S_{\lambda}\right) \rightarrow H\left(S_{\mu}\right)$.

To prove that the homology index sequence of an attractor-repeller pair is invariant under continuation, it is necessary to show that these isomorphisms define an isomorphism between the respective index sequences; i.e., that the following diagram commutes:

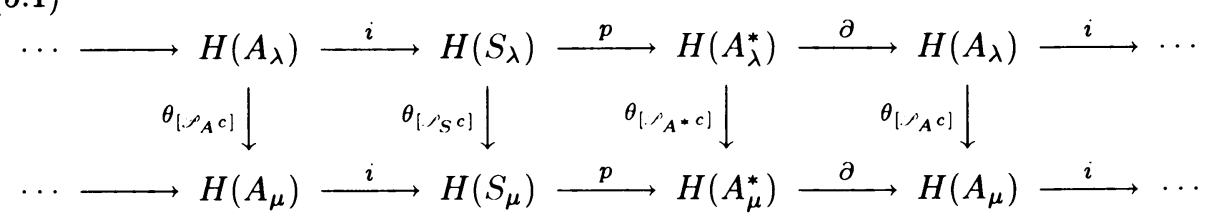


This follows from the work of Kurland [10]. His continuation results clearly carry over to our setting, and from Corollary 2.23 in [10] follows

Proposition 5.3. Let $\left(A_{\lambda}, A_{\lambda}^{*}\right)$ in $S_{\lambda},\left(A_{\mu}, A_{\mu}^{*}\right)$ in $S_{\mu}$, and $c$ be as above. If $\left(N_{2 \lambda}, N_{1 \lambda}, N_{0 \lambda}\right)$ and $\left(N_{2 \mu}, N_{1 \mu}, N_{0 \mu}\right)$ are index triples for the corresponding attractor-repeller pairs, then there exist homotopy equivalences $\theta, \theta^{\prime}$, and $\theta^{\prime \prime}$ inducing the homology isomorphisms $\theta_{\left[\mathscr{P}_{A} c\right]}, \theta_{\left[\mathscr{P}_{A^{*}}\right]}$, and $\theta_{\left[\mathscr{S}_{S} c\right]}$, respectively, and commuting in the following diagram:

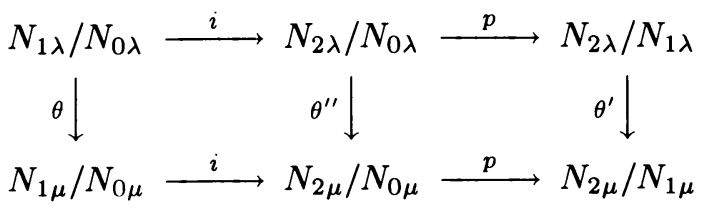

By the naturality of the long exact homology sequence, passing to homology in (5.2) yields the commutativity of diagram (5.1).

Assume that $M_{\lambda}=\left\{M_{\lambda}(\pi)\right\}_{\pi \in P}$ and $M_{\mu}=\left\{M_{\mu}(\pi)\right\}_{\pi \in P}$ are <-ordered Morse decompositions of isolated invariant sets $S_{\lambda} \subset X_{\lambda}$ and $S_{\mu} \subset X_{\mu}$, respectively. Denote the associated admissible orderings of $M_{\lambda}$ and $M_{\mu}$ by $<_{\lambda}$ and $<_{\mu}$, respectively. We have the following continuation theorem for homology index braids of admissible orderings of Morse decompositions.

THEOREM 5.4. If the admissible orderings $<_{\lambda}$ and $<_{\mu}$ are related by continuation, then $\mathscr{H}\left(<_{\lambda}\right)$ and $\mathscr{H}\left(<_{\mu}\right)$, the homology index braids of the admissible orderings, are isomorphic.

PROOF. If $c$ is a path in $\mathscr{M}_{<}$from $\times_{\pi \in P} M_{\lambda}(\pi) \times S_{\lambda}$ to $\times_{\pi \in P} M_{\mu}(\pi) \times S_{\mu}$, then for each $I \in I(<), \mathscr{P}_{I} c$ is a path in $\mathscr{S}$ from $M_{\lambda}(I)$ to $M_{\mu}(I)$. By Theorem 5.1, there exist isomorphisms between homology indices, $\theta(I): H_{\lambda}(I) \rightarrow H_{\mu}(I)$ for each $I \in I(<)$. Define $\theta=\{\theta(I) \mid I \in I(<)\}$. To show that $\theta$ is an isomorphism from $\mathscr{H}\left(<_{\lambda}\right)$ to $\mathscr{H}\left(<_{\mu}\right)$, it is necessary to show that the following diagram commutes for each $(I, J) \in I_{2}(<)$ :

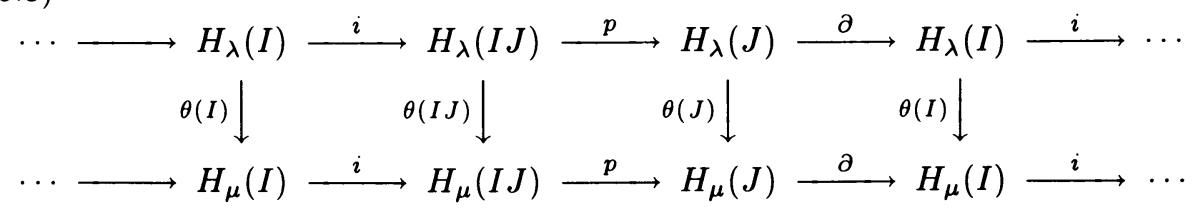

$\mathscr{P}_{I, J} c$ is a path in $\mathscr{A} \mathscr{R}$ from $\left(M_{\lambda}(I), M_{\lambda}(J), M_{\lambda}(I J)\right)$ to $\left(M_{\mu}(I), M_{\mu}(J), M_{\mu}(I J)\right)$; therefore, the corresponding homology index sequences are isomorphic via the isomorphisms associated to the paths $\mathscr{P}_{A} \mathscr{P}_{I, J} c, \mathscr{P}_{A^{*}} \mathscr{P}_{I, J} c$, and $\mathscr{P}_{S} \mathscr{P}_{I, J} c$ in $\mathscr{S}$. By the commutativity of diagrams 4.2 a-c , it follows that these isomorphisms are the isomorphisms $\theta(I), \theta(J)$, and $\theta(I J)$ defined above. Thus, diagram 5.3 commutes, and $\theta$ defines an isomorphism from $\mathscr{H}\left(<_{\lambda}\right)$ to $\mathscr{H}\left(<_{\mu}\right)$.

Let $C=\{C \Delta(\pi)\}_{\pi \in P}$ be a collection of graded modules. The following global continuation theorem for $(C$-) connection matrices of admissible orderings of Morse decompositions follows directly from Proposition 1.5.A and Theorem 5.4.

THEOREM 5.5. If the admissible orderings $<_{\lambda}$ of $M_{\lambda}$ and $<_{\mu}$ of $M_{\mu}$ are related by continuation, then $\mathscr{C} \mathscr{M}\left(<_{\lambda}, C\right)=\mathscr{C} \mathscr{M}\left(<_{\mu}, C\right)$ and $\mathscr{C} \mathscr{M}\left(<_{\lambda}\right)=\mathscr{C} \mathscr{M}\left(<_{\mu}\right)$. 
The following corollary of Theorem 5.5 describes the relationship between $(C$-)connection matrices of Morse decompositions if the flow ordering of one Morse decomposition continues to an admissible ordering of another. Our local connection matrix continuation result (Theorem 5.7, below) is a consequence of this result.

COROLLARY 5.6. If the flow ordering of $M_{\lambda}$ is related by continuation to an admissible ordering of $M_{\mu}$, then

$$
\mathscr{C} \mathscr{M}\left(M_{\mu}, C\right) \subset \mathscr{C} \mathscr{M}\left(M_{\lambda}, C\right)
$$

and

$$
\mathscr{C} \mathscr{M}\left(M_{\mu}\right) \subset \mathscr{C} \mathscr{M}\left(M_{\lambda}\right) .
$$

PROOF. Let $<_{F}$ denote the flow ordering of $M_{\lambda}$, and assume that $<_{F}$ is related by continuation to an admissible ordering $<$ of $M_{\mu}$. Then by Theorem 5.5 , $\mathscr{C} \mathscr{M}(<, C)=\mathscr{C} \mathscr{M}\left(<_{F}, C\right)$ and $\mathscr{C} \mathscr{M}(<)=\mathscr{C} \mathscr{M}\left(<_{F}\right)$. The $(C$-) connection matrices of a Morse decomposition are the $(C$-)connection matrices of the flow ordering of the Morse decomposition, and any admissible ordering of a Morse decomposition is an extension of its flow ordering. Therefore, with the aid of Proposition 1.5.B, it follows that $\mathscr{C} \mathscr{M}\left(M_{\mu}, C\right) \subset \mathscr{C} \mathscr{M}\left(M_{\lambda}, C\right)$ and $\mathscr{C} \mathscr{M}\left(M_{\mu}\right) \subset \mathscr{C} \mathscr{M}\left(M_{\lambda}\right)$.

The local continuation of Morse decompositions is established in $\S 4$. The following local continuation theorem for connection matrices of Morse decompositions states that $(C$-)connection matrices of a Morse decomposition may be lost, but not gained, under local continuation. In this sense, the collection of $(C$-)connection matrices of a Morse decomposition is upper semicontinuous over the space of Morse decompositions (and over the parameter space $\Lambda$ ).

THEOREM 5.7. There exists a neighborhood $W$ of $\lambda$ in $\Lambda$ such that if $\mu \in W$, then $M_{\lambda}$ is related by continuation to a Morse decomposition $M_{\mu}$ of an isolated invariant set in $X_{\mu}$, and for such $M_{\mu}, \mathscr{C} \mathscr{M}\left(M_{\mu}, C\right) \subset \mathscr{C} \mathscr{M}\left(M_{\lambda}, C\right)$ and $\mathscr{C} \mathscr{M}\left(M_{\mu}\right) \subset$ $\mathscr{C} \mathscr{M}\left(M_{\lambda}\right)$.

PROOF. Let $<_{F}$ be the flow ordering of $M_{\lambda}$. By Theorem 4.13, there is a homeomorphism between a neighborhood $V$ of $\lambda$ in $\Lambda$ and a neighborhood of $\times_{\pi \in P} M_{\lambda}(\pi) \times S_{\lambda}$ in $\mathscr{M}_{<_{F}}$. By the local path connectivity of $\Lambda$, there is a path connected neighborhood $W$ of $\lambda$ in $V$. For each $\mu \in W$, the flow ordering of $M_{\lambda}$ is related by continuation to an admissible ordering of a Morse decomposition $M_{\mu}$ of an isolated invariant set in $X_{\mu}$. By Corollary 5.6, $\mathscr{C} \mathscr{M}\left(M_{\mu}, C\right) \subset \mathscr{C} \mathscr{M}\left(M_{\lambda}, C\right)$ and $\mathscr{C} \mathscr{M}\left(M_{\mu}\right) \subset \mathscr{C} \mathscr{M}\left(M_{\lambda}\right)$.

6. An example. We consider the following family of ordinary differential equations parameterized by the variable $\theta>0$ :

$$
\dot{x}=y, \quad \dot{y}=\theta y-x\left(x-\frac{1}{3}\right)(1-x)
$$

In Figure 6.1, the complete set of bounded solutions for these equations, $S_{\theta}$, is shown (along with some nearby orbits) for values of $\theta$ near 0 and for values of $\theta$ large. For all $\theta>0$, the set of bounded solutions is an isolated invariant set, and if $M_{\theta}(1), M_{\theta}(2), M_{\theta}(3)$ are the points $(1 / 3,0),(0,0),(1,0)$, respectively, in the plane, then $M_{\theta}:=\left\{M_{\theta}(i)\right\}$ is a Morse decomposition of $S_{\theta}$. 
$0<\theta \ll 1$

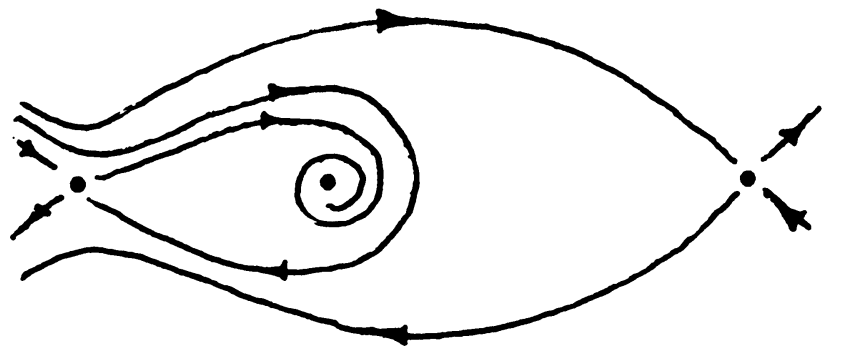

$\theta \gg 1$

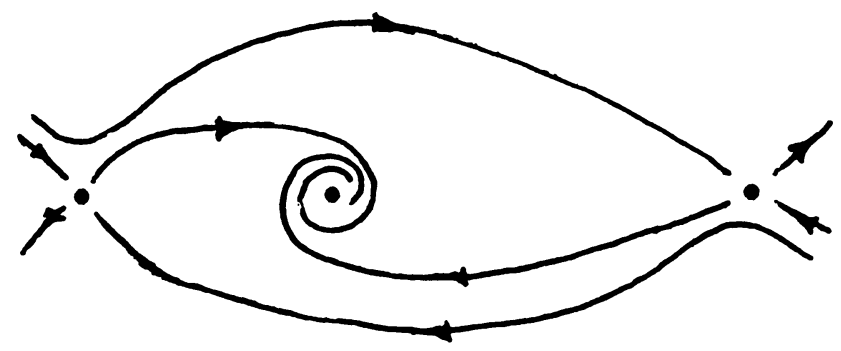

$M_{\theta}(2)$

$M_{\theta}(1)$

$M_{\theta}(3)$

FIGURE 6.1

Qualitatively, the flows in Figure 6.1 can be depicted as follows:
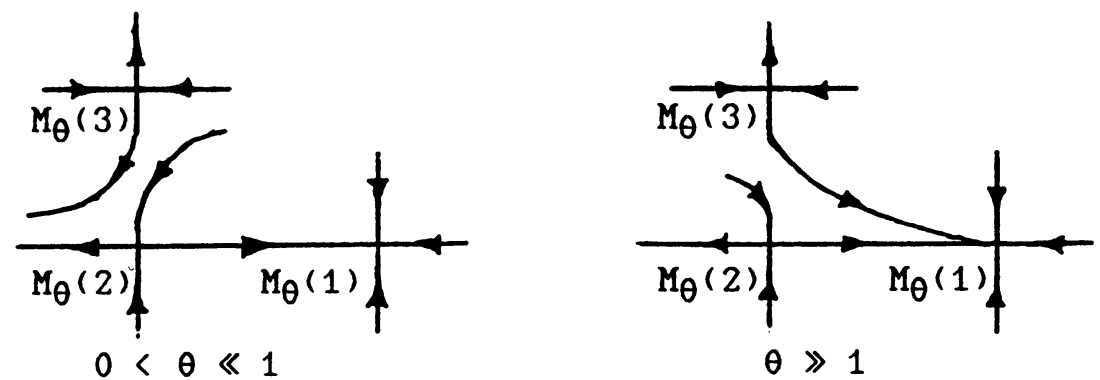

We use the connection matrix continuation theory to prove that there exists a parameter value $\theta^{*}$ such that $C\left(M_{\theta^{*}}(3), M_{\theta^{*}}(2)\right) \neq \varnothing$. Homology indices are computed with coefficients in $\mathrm{Z}_{2}$. Assume $\Delta \in \mathscr{C} \mathscr{M}\left(M_{\theta}\right)$ for some $\theta>0$. The homology index of each $M_{\theta}(i)$ is finitely generated; therefore, $\Delta$ is strictly upper triangular relative to the flow ordering (see [5]). Since $1<2<3$ defines an admissible ordering for all $\theta$, it follows that $\Delta_{j, i}=0$ if $i<j$. The homology indices of $M_{\theta}(3)$ and $M_{\theta}(2)$ are both nontrivial only in dimension one. Therefore, since each entry in $\Delta$ has degree -1 , we must have $\Delta_{2,3}=0$. For all $\theta>0$, the homology index of $M_{\theta}(12)$ is trivial; this implies (see [5]) that $\Delta_{1,2}$ is an isomorphism. 
It follows that if $\Delta \in \mathscr{C} \mathscr{M}\left(M_{\theta}\right)$ for some $\theta>0$, then $\Delta$ is in the form

$$
\left(\begin{array}{ccc}
0 & \approx & * \\
0 & 0 & 0 \\
0 & 0 & 0
\end{array}\right)
$$

where $\approx$ indicates an entry that is an isomorphism and $*$ denotes an entry that is either trivial or an isomorphism.

If $0<\theta \ll 1$, then $M_{\theta}(3)$ and $M_{\theta}(1)$ are noncomparable under the flow ordering, and therefore the upper triangularity of $\Delta$ requires $\Delta_{1,3}=0$. So, in this case, $\mathscr{C} \mathscr{M}\left(M_{\theta}\right)$ contains a single matrix, and it is in the form

$$
\left(\begin{array}{ccc}
0 & \approx & 0 \\
0 & 0 & 0 \\
0 & 0 & 0
\end{array}\right)
$$

If $\theta \gg 1$, then $M_{\theta}(1)$ and $M_{\theta}(3)$ are adjacent under the flow ordering and the homology index of $M_{\theta}(13)$ is trivial. Thus, as with $\Delta_{1,2}$, it follows that $\Delta_{1,3}$ is an isomorphism. Therefore, if $\theta \gg 1$, then $\mathscr{C} \mathscr{M}\left(M_{\theta}\right)$ also contains a single matrix, and it is in the form

$$
\left(\begin{array}{ccc}
0 & \approx & \approx \\
0 & 0 & 0 \\
0 & 0 & 0
\end{array}\right)
$$

Let $\mathscr{C} \mathscr{M}_{1}$ and $\mathscr{C} \mathscr{M}_{2}$ respectively denote the first and second collections of connection matrices described above, and for $i=1,2$, define $\theta_{i}=\left\{\theta \in(0, \infty) \mid \mathscr{C} \mathscr{M}\left(M_{\theta}\right)=\right.$ $\left.\mathscr{C} \mathscr{M}_{i}\right\} . \theta_{1}$ and $\theta_{2}$ are disjoint and both nonempty. The local connection matrix continuation theorem (Theorem 5.7) and the connection matrix existence theorem (see [5]) imply that each $\theta_{i}$ is open. By the connectivity of $(0, \infty)$, there exists $\theta^{*} \in(0, \infty) \backslash\left(\theta_{1} \cup \theta_{2}\right)$.

For such $\theta^{*}$, it follows that $\mathscr{C} \mathscr{M}\left(M_{\theta^{*}}\right)$ contains both of the matrices

$$
\left(\begin{array}{ccc}
0 & \approx & 0 \\
0 & 0 & 0 \\
0 & 0 & 0
\end{array}\right) \text { and }\left(\begin{array}{ccc}
0 & \approx & \approx \\
0 & 0 & 0 \\
0 & 0 & 0
\end{array}\right)
$$

$\Delta_{1,3}$ is nontrivial in the second matrix; therefore $1<_{F} 3$ in the flow ordering of $M_{\theta^{*}}$. We claim that $M_{\theta^{*}}$ (3) and $M_{\theta^{*}}$ (1) are not adjacent under the flow ordering. Suppose they are; then, as in the calculations for $\theta$ small and large, the $\Delta_{1,3}$ connection matrix entry is uniquely determined and is either trivial or an isomorphism. However, $\Delta_{1,3}$ is not uniquely determined, proving the claim. This implies that $1<_{F} 2<_{F} 3$, and the latter relation yields $C\left(M_{\theta^{*}}(3), M_{\theta^{*}}(2)\right) \neq \varnothing$.

In [5] this result is proved using a transition system. That technique involves putting a flow on the parameter space and then analyzing the flow on the product (phase $\times$ parameter) space. The method used here has the advantage that an artificial flow need not be put on the parameter space to carry out the analysis of the parameterized family of flows. This technique is generalized in [7] where we use the continuation theory and similarity transformations on connection matrices to study bifurcations of behavior in parameterized families of flows. The results in [7] yield an alternative technique to the transition systems studied in [12-17] and, as in the above example, at least have the advantage that the parameterized families can be studied without the need to add a flow on the parameter space. 


\section{REFERENCES}

1. C. Conley, Isolated invariant sets and the Morse index, CBMS Regional Conf. Ser. 38, Amer. Math. Soc., Providence, R. I., 1980.

2. C. Conley and E. Zehnder, Morse-type index theory for flows and perindic solutions for Hamiltonian equations, Comm. Pure Appl. Math. 37 (1984), 207-253.

3. R. Franzosa, Index filtrations and connection matrices for partially ordered Morse decompositions, $\mathrm{Ph}$. D. dissertation, Univ. of Wisconsin-Madison, 1984.

4. __ Index filtrations and the homology index braid for partially ordered Morse decompositions, Trans. Amer. Math. Soc. 298 (1986), 193-213.

5. _ , The connection matrix theory for Morse decompositions, Trans. Amer. Math. Soc. (to appear).

6. R. Franzosa and K. Mischaikow, The connection matrix theory for semiflows on metric spaces, J. Differential Equations 71 (1988), 270-287.

7. __ Connections in parameterized families of flows via similarity transformations on connection matrices (in preparation).

8. H. Kurland, The Morse index of an isolated invariant set is a connected simple system, J. Differential Equations 42 (1981).

9. —_, Homotopy invariants of repeller-attractor pairs. I. The Püppe sequence of an $r$-a pair, J. Differential Equations 46 (1982).

10. —_, Homotopy invariants of repeller-attractor pairs. II. Continuation of $r$-a pairs, J. Differential Equations 49 (1983).

11. C. McCord, Mappings and homological properties in the Conley index theory, $\mathrm{Ph}$. $\mathrm{D}$. dissertation, Univ. of Wisconsin-Madison, 1986.

12. K. Mischaikow, Classification of traveling wave solutions of reaction-diffusion systems, Brown Univ., LCDS \#86-5, 1985.

13. __ Homoclinic orbits in Hamiltonian systems and heteroclinic orbits in gradient and gradientlike systems, Brown. Univ., LCDS \#86-33, 1986.

14. __ Existence of generalized homoclinic orbits for one parameter families of flows, Brown Univ., LCDS \#86-42, 1986.

15. J. Reineck, The connection matrix and the classification of flows arising from ecological models, $\mathrm{Ph}$. D. dissertation, Univ. of Wisconsin-Madison, 1985.

16. _ Connecting orbits in one parameter families of flows, preprint.

17. __ Travelling wave solutions to a gradient system, preprint.

18. D. Salamon, Connected simple systems and the Conley index of isolated invariant sets, Trans. Amer. Math. Soc. 291 (1985), 1-41.

19. J. Smoller, Shock waves and reaction-diffusion equations, Springer-Verlag, New York, 1983.

20. E. Spanier, Algebraic topology, McGraw-Hill, 1966.

\section{Department of Mathematics, University of Maine, Orono, Maine 04469}

